\title{
A quadratic refinement of the Grothendieck-Lefschetz-Verdier trace formula
}

\author{
MARC HOYOIS
}

\begin{abstract}
We prove a trace formula in stable motivic homotopy theory over a general base scheme, equating the trace of an endomorphism of a smooth proper scheme with the "Euler characteristic integral" of a certain cohomotopy class over its scheme of fixed points. When the base is a field and the fixed points are étale, we compute this integral in terms of Morel's identification of the ring of endomorphisms of the motivic sphere spectrum with the Grothendieck-Witt ring. In particular, we show that the Euler characteristic of an étale algebra corresponds to the class of its trace form in the Grothendieck-Witt ring.
\end{abstract}

14F42; 47H10, 11E81

\section{Introduction and examples}

Let $k$ be a field, $X$ a smooth proper $k$-scheme and $f: X \rightarrow X$ a $k$-morphism. The Grothendieck-Lefschetz-Verdier trace formula, originally proved by Grothendieck in [11, Exposé III, Section 4], identifies the trace of the action of $f$ on the $\ell$-adic cohomology of $X$ with the integral of a cohomology class on the scheme of fixed points $X^{f}$. In the special case where $X^{f}$ is étale over $k$, the trace formula takes the following simple form:

Theorem 1.1 Let $k$ be a field, $X$ a smooth and proper $k$-scheme and $f: X \rightarrow X$ a $k$-morphism with étale fixed points. Then

$$
\sum_{i}(-1)^{i} \operatorname{tr}\left(f^{*} \mid H_{\ell}^{i}(\bar{X})\right)=\sum_{x \in X^{f}}[\kappa(x): k],
$$

where $\bar{X}$ is the pullback of $X$ to an algebraic closure of $k, \ell \neq$ char $k$ is a prime number and $H_{\ell}^{*}(-)$ is $\ell$-adic cohomology with coefficients in $\mathbb{Q}_{\ell}$.

The trace formula is thus an equality between two integers associated with $f$. The starting point of the present article is the observation that the left-hand side of the trace formula has a canonical refinement to an element of the Grothendieck-Witt ring 
$\mathrm{GW}(k)$ of the field $k$. To explain why, we need to recall some facts from stable motivic homotopy theory.

Let $\mathrm{Sm}_{k}$ be the category of smooth separated schemes of finite type over $k$. Consider the functor

$$
C_{*}^{\ell}: \operatorname{Sm}_{k} \rightarrow \widehat{\mathrm{D}}\left(\operatorname{Spec} k_{\mathrm{e} t}, \mathbb{Z}_{\ell}\right)
$$

that sends $p: X \rightarrow \operatorname{Spec} k$ to the $\ell$-adic sheaf $p ! p^{!} \mathbb{Z}_{\ell}$ on $\operatorname{Spec} k_{\text {ét }}$ (here $\widehat{\mathrm{D}}\left(B_{\text {ét }}, \mathbb{Z}_{\ell}\right)$ is the $\infty$-categorical limit over $n \geq 0$ of the derived categories $\left.\mathrm{D}\left(B_{\text {et }}, \mathbb{Z} / \ell^{n}\right)\right)$. By standard properties of $\ell$-adic cohomology and the definition of the stable motivic homotopy category $\mathrm{SH}(k)$, there is a canonical factorization

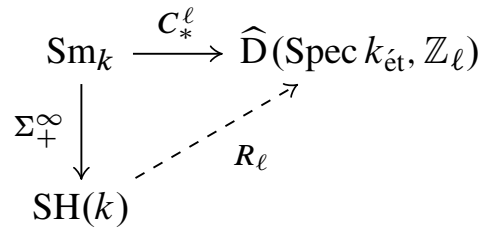

where $R_{\ell}$ is a symmetric monoidal functor. The functor $\Sigma_{+}^{\infty}$ satisfies a generalized version of Poincaré duality, which asserts in particular that, if $X$ is smooth and proper over $k, \Sigma_{+}^{\infty} X$ is strongly dualizable. Thus, if $f: X \rightarrow X$ is a $k$-morphism, $\Sigma_{+}^{\infty} f$ has a trace $\operatorname{tr}\left(\Sigma_{+}^{\infty} f\right)$ which is an endomorphism of the motivic sphere spectrum $\mathbf{1}_{k} \in \mathrm{SH}(k)$. Since symmetric monoidal functors commute with traces, $R_{\ell}\left(\operatorname{tr}\left(\Sigma_{+}^{\infty} f\right)\right)=$ $\operatorname{tr}\left(R_{\ell}\left(\Sigma_{+}^{\infty} f\right)\right)=\operatorname{tr}\left(C_{*}^{\ell} f\right)$, and it is clear that $\operatorname{tr}\left(C_{*}^{\ell} f\right)$ equals the alternating sum appearing in Theorem 1.1.

Recall that $\mathrm{GW}(k)$ is the group completion of the semiring of isomorphism classes of nondegenerate symmetric bilinear forms over $k$ (or equivalently of nondegenerate quadratic forms if char $k \neq 2$ ). Associating to such a form the rank of its underlying vector space defines a ring homomorphism

$$
\mathrm{rk}: \mathrm{GW}(k) \rightarrow \mathbb{Z}
$$

which is an isomorphism if and only if $k$ is quadratically closed. Given $u \in k^{\times}$, we denote by $\langle u\rangle$ the class of the symmetric bilinear form $k \times k \rightarrow k,(a, b) \mapsto u a b$. These basic classes generate $\mathrm{GW}(k)$ as a group. A fundamental result of Morel ${ }^{1}$ states that there is a natural isomorphism

$$
\mathrm{GW}(k) \simeq \operatorname{End}\left(\mathbf{1}_{k}\right) .
$$

\footnotetext{
${ }^{1}$ This result is proved by Morel in [20] under the assumption that $k$ is perfect. However, Morel actually computes the Nisnevich sheaf on $\mathrm{Sm}_{k}$ associated with the presheaf $X \mapsto\left[\Sigma_{+}^{\infty} X, \mathbf{1}_{k}\right]$, and combining this stronger result with the base change arguments from [13, Appendix A] allows us to remove the assumption on $k$.
} 
To describe Morel's isomorphism, we first consider a more general construction. Suppose that $V$ is a vector bundle over a scheme $X$ and that $\phi: V \stackrel{\sim}{\rightarrow} V$ is a linear automorphism of $V$. The vector bundle $V$ induces a self-equivalence $\Sigma^{V}$ of $\operatorname{SH}(X)$, which can be informally described as "smash product with the sphere bundle of $V$ ". The composition

$$
\mathbf{1}_{X} \simeq \Sigma^{-V} \Sigma^{V} \mathbf{1}_{X} \stackrel{\Sigma^{\phi}}{\longrightarrow} \Sigma^{-V} \Sigma^{V} \mathbf{1}_{X} \simeq \mathbf{1}_{X}
$$

is an automorphism of the motivic sphere spectrum over $X$, which we denote by $\langle\phi\rangle .^{2}$ The isomorphism (1-2) is then given by sending $\langle u\rangle$ to $\langle u\rangle$, viewing $u \in k^{\times}$as a linear automorphism of $\mathbb{A}_{k}^{1}$.

Putting all these facts together, we can identify $\operatorname{tr}\left(\Sigma_{+}^{\infty} f\right)$ with a lift of the integer $\operatorname{tr}\left(C_{*}^{\ell} f\right)$ to $\mathrm{GW}(k)$. It is then natural to ask whether the right-hand side of the Grothendieck-Lefschetz-Verdier trace formula also lifts to $\mathrm{GW}(k)$, ie whether there exist fixed-point indices $i(f, x) \in \mathrm{GW}(k)$, of rank $[\kappa(x): k]$, such that

$$
\operatorname{tr}\left(\Sigma_{+}^{\infty} f\right)=\sum_{x \in X^{f}} i(f, x)
$$

An affirmative answer is given in Corollary 1.10 below. ${ }^{3}$ It is a consequence of some more general results which we now discuss.

We consider an arbitrary base scheme $B$. If $X$ is a smooth $B$-scheme such that $\Sigma_{+}^{\infty} X \in \mathrm{SH}(B)$ is strongly dualizable, eg a smooth proper $B$-scheme, we write

$$
\chi(X)=\operatorname{tr}\left(\Sigma_{+}^{\infty} \mathrm{id}_{X}\right) \in \operatorname{End}\left(\mathbf{1}_{B}\right)
$$

for its Euler characteristic in $\mathrm{SH}(B)$. More generally, if $\omega$ is an endomorphism of $\mathbf{1}_{X}$ in $\mathrm{SH}(X)$, we define

$$
\int_{X} \omega d \chi=\operatorname{tr}\left(p_{\sharp} \omega\right) \in \operatorname{End}\left(\mathbf{1}_{B}\right),
$$

where $p: X \rightarrow B$ is the structure map and $p_{\sharp}: \mathrm{SH}(X) \rightarrow \mathrm{SH}(B)$ is left adjoint to the base change functor $p^{*}$. Note that, by this adjunction, an endomorphism of $\mathbf{1}_{X}$ is the same thing as a morphism $\Sigma_{+}^{\infty} X \rightarrow \mathbf{1}_{B}$ in $\operatorname{SH}(B)$. The map $\omega \mapsto \int_{X} \omega d \chi$ is thus an End $\left(\mathbf{1}_{B}\right)$-linear functional on the algebra of $\mathbf{1}_{B}$-valued functions on $\Sigma_{+}^{\infty} X$, such that $\int_{X} 1 d \chi=\chi(X)$.

We can now state the main result of this paper. Let $X$ be a smooth $B$-scheme, $f: X \rightarrow X$ a $B$-morphism and $i: X^{f} \hookrightarrow X$ the inclusion of the scheme of fixed points of $f$. We say that $f$ has regular fixed points if:

\footnotetext{
${ }^{2}$ This construction is of course the algebro-geometric analog of the $J$-homomorphism.

${ }^{3}$ The existence of such a fixed-point formula was mentioned by Morel in [19, Remark 4.12(2)].
} 
- $X^{f}$ is smooth over $B$.

- The endomorphism of the conormal sheaf $\mathcal{N}_{i}$ induced by id $-i^{*}(d f)$ is an isomorphism.

Theorem 1.3 Let $X$ be a smooth and proper $B$-scheme and $f: X \rightarrow X$ a $B-$ morphism with regular fixed points. Then

$$
\operatorname{tr}\left(\Sigma_{+}^{\infty} f\right)=\int_{X^{f}}\langle\phi\rangle d \chi
$$

where $\phi$ is the automorphism of the conormal sheaf of the immersion $i: X^{f} \hookrightarrow X$ induced by $\mathrm{id}-i^{*}(d f)$.

Theorem 1.3 will be proved in Section 4. The following special case is worth recording:

Corollary 1.4 Let $X$ be a smooth and proper $B$-scheme and $f: X \rightarrow X$ a $B$ morphism. If $\operatorname{tr}\left(\Sigma_{+}^{\infty} f\right) \neq 0$, then $f$ has a fixed point.

Along the way we will observe that $\chi(X)=0$ if $\Omega_{X / B}$ has a nonvanishing global section (see Remark 4.7):

Theorem 1.5 Let $X$ be a smooth and proper $B$-scheme. If $\left[\Omega_{X / B}\right]=\left[\mathcal{O}_{X}\right]+[\mathcal{E}]$ in $K_{0}(X)$ for some locally free sheaf $\mathcal{E}$, then $\int_{X} \omega d \chi=0$ for all $\omega \in \operatorname{End}\left(\mathbf{1}_{X}\right)$.

The properness hypothesis in Theorems 1.3 and 1.5 is essential: there are many smooth $B$-schemes that become strongly dualizable in $\mathrm{SH}(B)$ without being proper (eg the complement of a smooth closed subscheme in a smooth proper scheme) but these theorems clearly do not extend to all such schemes.

Before giving examples, we make some general remarks on the notion of regular fixed points appearing in Theorem 1.3. Let $\Delta_{X} \subset X \times_{B} X$ be the diagonal and let $\Gamma_{f} \subset X \times_{B} X$ be the graph of $f$. It is clear that we have the following implications:

$\Gamma_{f}$ and $\Delta_{X}$ intersect transversely

$\Downarrow$

$f$ has regular fixed points

$\Downarrow$

$\Gamma_{f}$ and $\Delta_{X}$ intersect cleanly

(The last simply means that $X^{f}$ is smooth over $B$.) Moreover, both implications are strict: the transposition on $X \times_{B} X$ has regular fixed points if and only if multiplication 
by 2 on $\Omega_{X / B}$ is invertible. Even in the case of a transverse intersection, we will see in Example 1.7 below that $\int_{X}\langle\phi\rangle d \chi$ can depend on $\phi$. In particular, the trace of $\Sigma_{+}^{\infty} f$ is not determined by the derived fixed points of $f$, since the latter coincide with the underived fixed points when the intersection of $\Gamma_{f}$ and $\Delta_{X}$ is transverse. This is a significant difference between stable motivic homotopy and $\ell$-adic cohomology.

Example 1.6 (Fixed points of Frobenius) Let $q$ be a prime power, $X$ a smooth and proper $\mathbb{F}_{q}$-scheme and $f: X \rightarrow X$ the Frobenius endomorphism. Then

$$
X^{f} \simeq \coprod_{X\left(\mathbb{F}_{q}\right)} \operatorname{Spec} \mathbb{F}_{q}
$$

and $d f=0$. By Theorem $1.3, \operatorname{tr}\left(\Sigma_{+}^{\infty} f\right) \in \mathrm{GW}\left(\mathbb{F}_{q}\right)$ is simply the Euler characteristic of $X^{f}$, which is the number of $\mathbb{F}_{q}$-rational points of $X$ by additivity of the trace.

Example 1.7 (The Euler characteristic of $\mathbb{P}^{1}$ ) We can compute the Euler characteristic of projective space $\mathbb{P}^{n}$ by induction on $n$ using the cofiber sequence

$$
\Sigma_{+}^{\infty} \mathbb{P}^{n-1} \rightarrow \Sigma_{+}^{\infty} \mathbb{P}^{n} \rightarrow S^{\mathbb{A}^{n}}
$$

and the additivity of the trace (see May [17]). We find that

$$
\chi\left(\mathbb{P}^{n}\right)= \begin{cases}\chi\left(\mathbb{P}^{n-1}\right)+1 & \text { if } n \text { is even, } \\ \chi\left(\mathbb{P}^{n-1}\right)+\tau & \text { if } n \text { is odd, }\end{cases}
$$

where $\tau \in \operatorname{End}\left(\mathbf{1}_{B}\right)$ is the desuspension of the transposition $S^{\mathbb{A}^{1}} \wedge S^{\mathbb{A}^{1}} \simeq S^{\mathbb{A}^{1}} \wedge S^{\mathbb{A}^{1}}$. If $B$ is the spectrum of a field $k$, it is well-known that $\tau$ corresponds to $\langle-1\rangle \in \mathrm{GW}(k)$. As a consistency test, we use Theorem 1.3 to show that the Euler characteristic of the projective line $\mathbb{P}^{1}$ over $k$ is the hyperbolic form $\langle 1,-1\rangle \in \mathrm{GW}(k)$. Since an odd-degree extension of finite fields induces an isomorphism on Grothendieck-Witt rings, we may assume without loss of generality that $k$ has at least 4 elements. Choose $a \in k^{\times}$with $a^{2} \neq 1$ and let $f$ be the automorphism of $\mathbb{P}^{1}$ given by $[x: y] \mapsto\left[a^{2} x: y\right]$. A homotopy between the matrices

$$
\left(\begin{array}{ll}
1 & 0 \\
0 & 1
\end{array}\right) \text { and }\left(\begin{array}{cc}
a & 0 \\
0 & a^{-1}
\end{array}\right)
$$

in $\operatorname{SL}_{2}(k)$ induces a homotopy between $\operatorname{id}_{\mathbb{P}^{1}}$ and $f$, so that $\chi\left(\mathbb{P}^{1}\right)=\operatorname{tr}\left(\Sigma_{+}^{\infty} f\right)$. We have

$$
\left(\mathbb{P}^{1}\right)^{f}=\{0, \infty\}
$$

${ }^{4}$ Here we use the following fact: if $\mathcal{C}$ is a symmetric monoidal category and $L \in \mathcal{C}$ is $\otimes$-invertible, then $\chi(L) \in \operatorname{End}(\mathbf{1})$ corresponds to the transposition under the canonical isomorphism $\operatorname{End}(L \otimes L) \simeq \operatorname{End}(\mathbf{1})$. We leave the elementary proof to the reader. 
(a disjoint union of two copies of $\operatorname{Spec}(k)), d f_{0}=a^{2}$, and $d f_{\infty}=a^{-2}$. Thus, the endomorphism id $-i^{*}(d f)$ of $i^{*}\left(\Omega_{\mathbb{P}^{1}}\right) \simeq \mathcal{N}_{i}$ is multiplication by $1-a^{2}$ at 0 and by $1-a^{-2}$ at $\infty$. By Theorem 1.3 , the trace of $f$ is $\left\langle 1-a^{2}, 1-a^{-2}\right\rangle=\langle 1,-1\rangle$, as expected.

Example 1.8 (Relations in the endomorphism ring of the motivic sphere spectrum) The fact that $\operatorname{tr}\left(\Sigma_{+}^{\infty} f\right)$ is an invariant of the homotopy class of $f$ produces interesting relations in the ring $\operatorname{End}\left(\mathbf{1}_{B}\right)$. For example, if $k$ is a field and $a_{0}, \ldots, a_{n} \in k^{\times}$are $n+1$ distinct elements whose product is 1 , then the endomorphism $\left[x_{0}: \ldots: x_{n}\right] \mapsto$ $\left[a_{0} x_{0}: \ldots: a_{n} x_{n}\right]$ of $\mathbb{P}^{n}$ over $k$ is homotopic to the identity. It follows that its trace, which by Theorem 1.3 is the class

$$
\sum_{i=0}^{n} \prod_{j \neq i}\left\langle 1-a_{j} / a_{i}\right\rangle \in \mathrm{GW}(k),
$$

is independent of the choice of the elements $a_{i}$ and equals the Euler characteristic of $\mathbb{P}^{n}$.

Our proofs of Theorems 1.3 and 1.5 remain valid if the functor $B \mapsto \mathrm{SH}(B)$ is replaced by any motivic triangulated category in the sense of Cisinski-Déglise [5, Definition 2.4.45]. On the other hand, by the $\infty$-categorical universality of $\operatorname{SH}(B)$ for fixed $B$ established by Robalo [24], our theorems admit the following generalizations. Let $\mathcal{C}$ be a pointed symmetric monoidal presentable $\infty$-category and $F: \operatorname{Sm}_{B} \rightarrow \mathcal{C}$ a symmetric monoidal functor satisfying $\mathbb{A}^{1}$-homotopy invariance, Nisnevish descent, and $\mathbb{P}^{1}$-stability (ie the cofiber of $F(\infty) \rightarrow F\left(\mathbb{P}_{B}^{1}\right)$ is $\otimes$-invertible). Then $F$ sends smooth proper $B$-schemes to strongly dualizable objects and Theorems 1.3 and 1.5 are true with $\Sigma_{+}^{\infty}$ replaced by $F$. For example, when $B$ is a field and $F=C_{*}^{\ell}$, Theorem 1.3 recovers Theorem 1.1.

Finally, in Section 5, we will prove:

Theorem 1.9 Let $k \subset L$ be a finite separable field extension, $V$ a finite-dimensional vector space over $L$ and $\phi$ an automorphism of $V$. Then, modulo the isomorphism (1-2),

$$
\int_{L}\langle\phi\rangle d \chi=\operatorname{Tr}_{L / k}\langle\operatorname{det}(\phi)\rangle .
$$

Here $\operatorname{Tr}_{L / k}: \mathrm{GW}(L) \rightarrow \mathrm{GW}(k)$ is the Scharlau transfer associated with the field trace $\operatorname{Tr}_{L / k}: L \rightarrow k$, ie it sends a symmetric bilinear form $b: V \times V \rightarrow L$ to the form $\operatorname{Tr}_{L / k} \circ b: V \times V \rightarrow k$ of rank $[L: k] \operatorname{rk}(b)$. Note that we allow $k$ to have characteristic 2 or to be imperfect. Combining Theorems 1.3 and 1.9 gives the following result, which is a motivic version of the Lefschetz-Hopf theorem; see Dold [7, VII, Proposition 6.6]: 
Corollary 1.10 Let $k$ be a field, $X$ a smooth and proper $k$-scheme and $f: X \rightarrow X$ a $k$-morphism with étale fixed points. Then

$$
\operatorname{tr}\left(\Sigma_{+}^{\infty} f\right)=\sum_{x \in X^{f}} \operatorname{Tr}_{\kappa(x) / k}\left\langle\operatorname{det}\left(\mathrm{id}-d f_{x}\right)\right\rangle
$$

Example 1.11 (The Euler characteristic of $\mathbb{P}^{1}$, continued) Let $k$ be a field such that $\sqrt{-1} \notin k$. Consider the endomorphism $f: \mathbb{P}^{1} \rightarrow \mathbb{P}^{1}$ given by $[x: y] \mapsto[-y: x]$. It is again induced by a matrix in $\mathrm{SL}_{2}(k)$ and hence, as in Example 1.7, is homotopic to $\operatorname{id}_{\mathbb{P}^{1}}$. We have

$$
\left(\mathbb{P}^{1}\right)^{f} \simeq \operatorname{Spec} k(i),
$$

where $i$ is a square root of -1 . Moreover, $d f_{i}$ is multiplication by $i^{-2}=-1$. The fixed-point index of $f$ at $i$ is therefore

$$
\operatorname{Tr}_{k(i) / k}\langle 1-(-1)\rangle=\langle 4,-4\rangle=\langle 1,-1\rangle \in \mathrm{GW}(k) .
$$

As predicted by Corollary 1.10, this coincides with the Euler characteristic of $\mathbb{P}^{1}$ computed in Example 1.7.

Conventions The following conventions are in force throughout, except in Appendix C:

- All schemes are assumed to be coherent, ie quasi-compact and quasi-separated.

- Smooth and étale morphisms are assumed to be separated and of finite type.

See however Remark C.14.

Acknowledgements I thank Marc Levine and Jean Fasel for their interest in this project and for stimulating conversations about it. The first version of this paper was written while I was visiting the department of mathematics at the University of Duisburg-Essen and I would like to thank everyone there for their hospitality. Finally, I am immensely grateful to the anonymous referee whose report lead to considerable improvements to the original manuscript.

\section{Review of the formalism of six operations}

To prove Theorem 1.3, we will use the formalism of six operations $\left(f^{*}, f_{*}, f_{!}, f^{!}, \wedge\right.$ and Hom) in stable motivic homotopy theory developed by Ayoub in [3] and revisited by Cisinski and Déglise in [5]. In this section we briefly review the main features of this formalism and we introduce several pieces of notation that will be used throughout this paper. 
Remark 2.1 We do not insist that schemes be noetherian and of finite Krull dimension. We explain in Appendix $\mathrm{C}$ how to extend motivic homotopy theory and the formalism of six operations to arbitrary schemes.

For a scheme $B$, we denote by $\mathrm{SH}(B)$ the closed symmetric monoidal triangulated category of motivic spectra parametrized by $B$. The monoidal unit, monoidal product, monoidal symmetry and internal hom in $\mathrm{SH}(B)$ will be denoted by $\mathbf{1}_{B}, \wedge, \tau$ and Hom, respectively. We first give a description of the six operations which is independent of the specifics of the category $\mathrm{SH}(B)$.

To any morphism of schemes $f: Y \rightarrow X$ is associated an adjunction

$$
f^{*}: \mathrm{SH}(X) \rightleftarrows \mathrm{SH}(Y): f_{*},
$$

where $f^{*}$ is symmetric monoidal. If $f$ is smooth, $f^{*}$ also admits a left adjoint denoted by $f_{\sharp}$. If $f$ is separated of finite type, there is an exceptional adjunction

$$
f_{!}: \mathrm{SH}(Y) \rightleftarrows \mathrm{SH}(X): f^{!}
$$

and a natural transformation $f_{!} \rightarrow f_{*}$ which is an isomorphism when $f$ is proper. Each of the assignments $f \mapsto f^{*}, f_{*}, f_{!}, f^{!}, f_{\sharp}$ is part of a 2-functor on the category of schemes. In particular, every commutative triangle of schemes gives rise to various connection isomorphisms, such as $(g f)^{*} \simeq f^{*} g^{*}$, satisfying cocyle conditions. We will denote by $c$ any isomorphism which is a composition of such connection isomorphisms. To any cartesian square of schemes

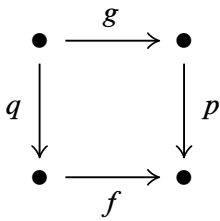

are associated several exchange transformations, such as

$$
\begin{aligned}
& E x_{*}^{*}: f^{*} p_{*} \rightarrow q_{*} g^{*}, \\
& E x^{* !}: g^{*} p^{!} \rightarrow q^{!} f^{*}, \\
& E x_{\sharp}^{*}: g_{\sharp} q^{*} \rightarrow p^{*} f_{\sharp} .
\end{aligned}
$$

To a morphism $f$ are also associated several projectors, such as

$$
\begin{aligned}
& P r_{*}^{*}: f_{*} E \wedge F \rightarrow f_{*}\left(E \wedge f^{*} F\right), \\
& \operatorname{Pr}^{* !}: f^{*} E \wedge f^{!} F \rightarrow f^{!}(E \wedge F), \\
& \operatorname{Pr}_{\sharp}^{*}: f_{\sharp}\left(E \wedge f^{*} F\right) \rightarrow f_{\sharp} E \wedge F .
\end{aligned}
$$


Each projector comes in left and right variants (for which we use the same symbol) related to one another via the monoidal symmetry $\tau$. There are also projectors involving the internal hom, but we will not need them. A crucial fact is that the transformations $E x_{!}^{*}, E x_{*}^{!}$and $P r_{!}^{*}$ are always isomorphisms. As we will see below, this generalizes the proper base change theorem ( $E x_{*}^{*}$ is an isomorphism when $p$ is proper), the smooth base change theorem ( $E x_{*}^{*}$ is an isomorphism when $f$ is smooth) and the projection formula $\left(\mathrm{Pr}_{*}^{*}\right.$ is an isomorphism when $f$ is proper).

If $i: Z \hookrightarrow X$ is a closed immersion with open complement $j: U \hookrightarrow X$, we have two localization cofiber sequences

$$
\begin{aligned}
& j_{!} j \stackrel{!}{\longrightarrow} \mathrm{id} \stackrel{\eta}{\longrightarrow} i_{*} i^{*}, \\
& i_{!} i^{!} \stackrel{\epsilon}{\longrightarrow} \mathrm{id} \stackrel{\eta}{\longrightarrow} j_{*} j^{*} .
\end{aligned}
$$

Moreover, the functors $i_{*} \simeq i_{!}, j_{\text {! }}$ and $j_{*}$ are fully faithful. We will denote by $\sigma: i^{!} \rightarrow i^{*}$ the natural transformation

$$
i^{!} \simeq \mathrm{id}^{*} i^{!} \stackrel{E x^{* !}}{\longrightarrow} \mathrm{id}^{!} i^{*} \simeq i^{*} .
$$

If $p: V \rightarrow X$ is a vector bundle with zero section $s$, the adjunction

$$
p_{\sharp} S_{*}: \mathrm{SH}(X) \rightleftarrows \mathrm{SH}(X): s^{!} p^{*}
$$

is a self-equivalence of $\mathrm{SH}(X)$, which we will denote by $\Sigma^{V} \dashv \Sigma^{-V}$. The functors $\Sigma^{V}$ and $\Sigma^{-V}$ will be called Thom transformations, or the $V$-suspension and $V$ desuspension functors, respectively. They are compatible with each of the operations $f^{*}, f_{*}, f_{\sharp}, f_{!}$and $f^{!}$in the following sense: there are canonical isomorphisms $f^{*} \Sigma^{V} \simeq \Sigma^{f^{*} V} f^{*}, \Sigma^{V} f_{*} \simeq f_{*} \Sigma^{f^{*} V}$ etc. They are also compatible with the monoidal structure, in the sense that $\Sigma^{V} E \wedge F \simeq \Sigma^{V}(E \wedge F)$ and $\Sigma^{-V} E \wedge F \simeq$ $\Sigma^{-V}(E \wedge F)$. In particular,

$$
\Sigma^{V} \simeq \Sigma^{V} \mathbf{1}_{X} \wedge(-) \text { and } \Sigma^{-V} \simeq \Sigma^{-V} \mathbf{1}_{X} \wedge(-) .
$$

If $\mathcal{M}$ is a locally free sheaf of finite rank on $X$, we will also denote by $\Sigma^{\mathcal{M}}$ and $\Sigma^{-\mathcal{M}}$ the functors $\Sigma^{\mathbb{V}(\mathcal{M})}$ and $\Sigma^{-\mathbb{V}(\mathcal{M})}$, where $\mathbb{V}(\mathcal{M})=\operatorname{Spec}(\operatorname{Sym}(\mathcal{M}))$ is the vector bundle on $X$ whose sheaf of sections is dual to $\mathcal{M}$.

If $f$ is smooth, there are canonical isomorphisms

$$
f_{!} \simeq f_{\sharp} \Sigma^{-\Omega_{f}} \quad \text { and } \quad f^{!} \simeq \Sigma^{\Omega_{f}} f^{*},
$$

where $\Omega_{f}$ is the sheaf of relative differentials of $f$. In particular, if $f$ is étale, $f_{!} \simeq f_{\#}$ and $f^{!} \simeq f^{*}$. At this point we see that the operations $f_{\sharp}, \Sigma^{V}$ and $\Sigma^{-V}$, which are 
not listed among the six operations, are expressible in terms of the latter as

$$
f_{\sharp} \simeq f_{!} \Sigma^{\Omega_{f}}, \quad \Sigma^{V} \simeq s^{*} p^{!}, \quad \Sigma^{-V} \simeq s^{!} p^{*} .
$$

The Thom transformations are functorial in monomorphisms of vector bundles (ie epimorphisms of locally free sheaves) as follows. Given a triangle

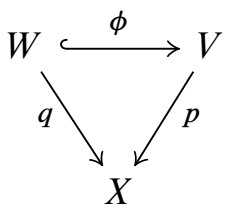

where $p$ and $q$ are vector bundles with zero sections $s$ and $t$ and where $\phi$ exhibits $W$ as a subbundle of $V$, we define $\Sigma^{\phi}: \Sigma^{W} \rightarrow \Sigma^{V}$ to be the composition

$$
t^{*} q^{!} \stackrel{c}{\simeq} t^{*} \phi^{!} p^{!} \stackrel{\sigma}{\rightarrow} t^{*} \phi^{*} p^{!} \stackrel{c}{\simeq} s^{*} p^{!}
$$

and we let $\Sigma^{-\phi}: \Sigma^{-V} \rightarrow \Sigma^{-W}$ be its mate, which is given by the same composition with stars and shrieks exchanged. In particular, a linear automorphism $\phi: V \stackrel{\sim}{\rightarrow} V$ induces an automorphism $\Sigma^{-V} \Sigma^{\phi}$ of the identity functor on $\mathrm{SH}(X)$, which we denote by $\langle\phi\rangle$.

For any short exact sequence

$$
0 \rightarrow W \rightarrow V \rightarrow U \rightarrow 0
$$

of vector bundles on $X$, the exchange transformation $E x^{* !}$ provides an isomorphism

$$
\Sigma^{V} \simeq \Sigma^{W} \Sigma^{U}
$$

which is natural with respect to monomorphisms of short exact sequence. The properties of these isomorphisms established in [3, Section 1.5] show that the association $V \mapsto \Sigma^{V}$ induces a morphism of Picard groupoids

$$
\Sigma^{(-)}: K(X) \rightarrow \operatorname{Aut}(\operatorname{SH}(X))
$$

from the $K$-theory groupoid of $X$ to the groupoid of self-equivalences of $\operatorname{SH}(X)$. In particular, the map $\phi \mapsto\langle\phi\rangle$ factors through a group homomorphism $K_{1}(X) \rightarrow$ Aut $\left(\operatorname{id}_{\mathrm{SH}(X)}\right)$.

Given a commutative triangle

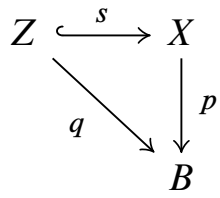


where $p$ and $q$ are smooth and $s$ is a closed immersion, we obtain a sequence of isomorphisms

$$
s^{!} p^{*} \simeq s^{!} \Sigma^{-\Omega_{p}} p^{!} \simeq \Sigma^{-s^{*}\left(\Omega_{p}\right)} s^{!} p^{!} \stackrel{c}{\simeq} \Sigma^{-s^{*}\left(\Omega_{p}\right)} q^{!} \simeq \Sigma^{-s^{*}\left(\Omega_{p}\right)} \Sigma^{\Omega_{q}} q^{*} \simeq \Sigma^{-\mathcal{N}_{s}} q^{*},
$$

where the last isomorphism is induced by the short exact sequence

$$
0 \rightarrow \mathcal{N}_{s} \rightarrow s^{*}\left(\Omega_{p}\right) \stackrel{d s}{\longrightarrow} \Omega_{q} \rightarrow 0 .
$$

The isomorphism $s^{!} p^{*} \simeq \Sigma^{-\mathcal{N}_{s}} q^{*}$ and its mate $p_{\sharp S_{*}} \simeq q_{\sharp} \Sigma^{\mathcal{N}_{s}}$ are called the purity isomorphisms and are denoted by $\Pi$. Although the purity isomorphism appears $a$ posteriori as a consequence of the formalism of six operations, it must be constructed "by hand" in both the approach of Ayoub and that of Cisinski-Déglise. We discuss the purity isomorphism further in Appendix A (where in particular we show that the constructions of Ayoub and of Cisinski-Déglise are equivalent).

Of course, all this data satisfies many coherence properties, of which an exhaustive list cannot easily be written down. Let us mention here one kind of coherence that we will use often. If $f$ is a smooth morphism (resp a proper morphism), then we may want to replace, in a given expression, occurrences of $f_{\text {! }}$ and $f^{!}$by $f_{\sharp} \Sigma^{-\Omega_{f}}$ and $\Sigma^{\Omega_{f}} f^{*}$ (resp. occurrences of $f_{!}$by $f_{*}$ ). Such replacements yield canonically isomorphic expressions and, under these canonical isomorphisms, any exchange transformation is transformed into another exchange transformation and any projector is transformed into another projector. For example, consider the cartesian square (2-2) and the exchange isomorphism $E x_{!}^{*}: p^{*} f_{!} \stackrel{\sim}{\rightarrow} g_{!} q^{*}$. If $f$ is smooth, then $q^{*}\left(\Omega_{f}\right) \simeq \Omega_{g}$ and the square

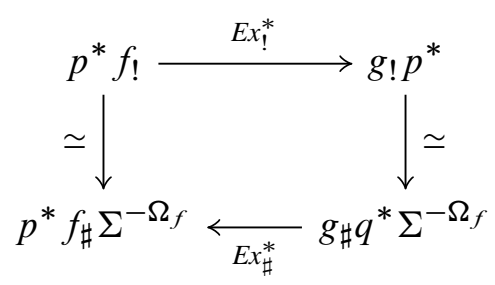

commutes, while the square

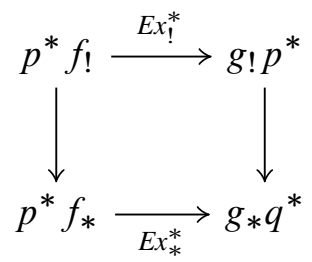


commutes for any $f$ (the vertical maps being isomorphisms when $f$ is proper). Similarly, when $f$ or $p$ is smooth, the exchange transformation $E x^{* !}$ transforms into the isomorphism $E x^{! !}$or $E x^{* *}$.

Let us now describe these functors more explicitly. For a scheme $B$, we denote by $\mathrm{Sm}_{B}$ the category of smooth $B$-schemes and by $\mathrm{H}_{(*)}(B)$ the (pointed) motivic homotopy category of $B$ (we refer to Appendix $C$ for the definitions in the generality considered here). We denote by

$$
\begin{aligned}
& \Sigma_{+}^{\infty}: \mathrm{H}(B) \rightarrow \mathrm{SH}(B), \\
& \Sigma^{\infty}: \mathrm{H}_{*}(B) \rightarrow \mathrm{SH}(B)
\end{aligned}
$$

the canonical symmetric monoidal functors, called stabilization functors. If $X \in \operatorname{Sm}_{B}$ and $U \hookrightarrow X$ is an open subscheme, $X / U$ is the quotient of the presheaf represented by $X$ by the presheaf represented by $U$, viewed as an object of $\mathrm{H}_{*}(B)$. If $V$ is a vector bundle on $X \in \mathrm{Sm}_{B}$, we denote its Thom space by

$$
\operatorname{Th}_{X}(V)=\frac{V}{V-X} \in \mathrm{H}_{*}(B) .
$$

If $V$ is a vector bundle over $B$ itself, we also write $S^{V}$ for $\operatorname{Th}_{B}(V)$ or for its stabilization $\Sigma^{\infty} \mathrm{Th}_{B}(V)$.

For $f: Y \rightarrow X$, the functor $f^{*}: \mathrm{SH}(X) \rightarrow \mathrm{SH}(Y)$ is induced by the base change functor $\mathrm{Sm}_{X} \rightarrow \mathrm{Sm}_{Y}$, so that

$$
f^{*} \Sigma_{+}^{\infty} U \simeq \Sigma_{+}^{\infty}\left(U \times_{X} Y\right) .
$$

If $f$ is smooth, the functor $f_{\sharp}$ is similarly induced by the forgetful functor $\operatorname{Sm}_{Y} \rightarrow \operatorname{Sm}_{X}$. In particular, if $p: X \rightarrow B$ is smooth, then

$$
\Sigma_{+}^{\infty} X \simeq p_{\sharp} p^{*} \mathbf{1}_{B} \simeq p_{!} p^{!} \mathbf{1}_{B} \in \mathrm{SH}(B) .
$$

If $i: Z \hookrightarrow B$ is a closed immersion with open complement $j: U \hookrightarrow B$ and if $X \in \operatorname{Sm}_{B}$, the localization cofiber sequence

$$
j_{\sharp} \Sigma_{+}^{\infty} X_{U} \rightarrow \Sigma_{+}^{\infty} X \rightarrow i_{*} \Sigma_{+}^{\infty} X_{Z}
$$

shows that

$$
i_{*} \Sigma_{+}^{\infty} X_{Z} \simeq \Sigma^{\infty}\left(X / X_{U}\right) .
$$

In particular, if $V$ is a vector bundle on $X$, then $\Sigma^{V} \mathbf{1}_{X} \simeq S^{V}$ and hence $\Sigma^{V} \simeq S^{V} \wedge(-)$. If $p: X \rightarrow B$ is smooth and $V$ is a vector bundle on $X$, we deduce that

$$
\Sigma^{\infty} \mathrm{Th}_{X}(V) \simeq p_{\sharp} \Sigma^{V} p^{*} \mathbf{1}_{B} \simeq p_{!} \Sigma^{V} p^{!} \mathbf{1}_{B} \in \mathrm{SH}(B) .
$$


Consider a commutative triangle

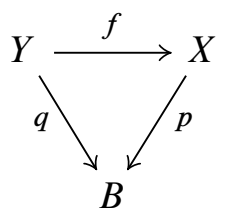

where $p$ and $q$ are smooth. Under the isomorphisms $\Sigma_{+}^{\infty} X \simeq p_{!} p^{!} \mathbf{1}_{B}$ and $\Sigma_{+}^{\infty} Y \simeq$ $q ! q ! \mathbf{1}_{B}$, the map $\Sigma_{+}^{\infty} f$ in $\mathrm{SH}(B)$ is given by the composition

$$
q ! q^{!} \mathbf{1}_{B} \stackrel{c}{\simeq} p_{!} f_{!} f^{!} p^{!} \mathbf{1}_{B} \stackrel{\epsilon}{\rightarrow} p_{!} p^{!} \mathbf{1}_{B}
$$

(this is [4, Lemme C.2]). More generally, suppose that $V$ and $W$ are vector bundles on $X$ and $Y$ and let $\phi: W \hookrightarrow f^{*} V$ be a monomorphism of vector bundles. Then the map of Thom spectra $\Sigma^{\infty} \mathrm{Th}_{Y}(W) \rightarrow \Sigma^{\infty} \mathrm{Th}_{X}(V)$ induced by $\phi$ is given by the composition

$q_{!} \Sigma^{W} q^{!} \mathbf{1}_{B} \stackrel{\Sigma^{\phi}}{\longrightarrow} q ! \Sigma^{f^{*} V} q^{!} \mathbf{1}_{B} \stackrel{c}{\simeq} p_{!} f_{!} \Sigma^{f^{*} V} f^{!} p^{!} \mathbf{1}_{B} \simeq p_{!} \Sigma^{V} f_{!} f^{!} p^{!} \mathbf{1}_{B} \stackrel{\epsilon}{\rightarrow} p_{!} \Sigma^{V} p^{!} \mathbf{1}_{B}$.

This is easily proved by considering the localization cofiber sequences defining $\operatorname{Th}_{Y}(W)$ and $\mathrm{Th}_{X}(V)$ and applying the previous result to the maps $W-Y \rightarrow V-X$ and $W \rightarrow V$.

Finally, given the triangle (2-4) with $p$ and $q$ smooth and $s$ a closed immersion, the purity isomorphism $\Pi: p_{\sharp} S_{*} \mathbf{1}_{X} \simeq q_{\sharp} \Sigma^{\mathcal{N}_{s}} \mathbf{1}_{X}$ is the stabilization of the unstable isomorphism

$$
\frac{X}{X-Z} \simeq \operatorname{Th}_{Z}\left(\mathbb{V}\left(\mathcal{N}_{S}\right)\right)
$$

in $\mathrm{H}_{*}(B)$ constructed by Morel and Voevodsky [21, Theorem 2.23].

\section{Duality in stable motivic homotopy theory}

Fix a base scheme $B$. Hu-Kriz [14, Appendix A] and Riou [23, Section 2] proved that smooth and projective $B$-schemes become strongly dualizable in $\mathrm{SH}(B)$. We will follow the latter reference and deduce this duality as an easy consequence of the formalism of six operations. We will then provide alternative descriptions of this duality that we will need in Section 4 and Section 5.

Recall that an object $A$ in a symmetric monoidal category $(\mathcal{C}, \otimes, \mathbf{1})$ is strongly dualizable if there exists an object $A^{\vee}$ and morphisms

$$
\text { coev: } \mathbf{1} \rightarrow A \otimes A^{\vee} \text { and } \quad \text { ev: } A^{\vee} \otimes A \rightarrow \mathbf{1}
$$


such that both compositions

$$
\begin{gathered}
A \simeq \mathbf{1} \otimes A \stackrel{\text { coev } \otimes \mathrm{id}}{\longrightarrow} A \otimes A^{\vee} \otimes A \stackrel{\mathrm{id} \otimes \mathrm{ev}}{\longrightarrow} A \otimes \mathbf{1} \simeq A, \\
A^{\vee} \simeq A^{\vee} \otimes \mathbf{1} \stackrel{\mathrm{id} \otimes \mathrm{coev}}{\longrightarrow} A^{\vee} \otimes A \otimes A^{\vee} \stackrel{\mathrm{ev} \otimes \mathrm{id}}{\longrightarrow} \mathbf{1} \otimes A^{\vee} \simeq A^{\vee}
\end{gathered}
$$

are the identity. When it exists, this data is unique up to a unique isomorphism. If objects $A$ and $A^{\vee}$ are given, then a choice of coevaluation and evaluation maps exhibiting $A^{\vee}$ as a strong dual of $A$ is equivalent to a choice of adjunction between $A^{\vee} \otimes(-)$ and $A \otimes(-)$. The counit and unit of such an adjunction determine the evaluation and the coevaluation, respectively. If $A \in \mathcal{C}$ is strongly dualizable and $f: A \rightarrow A$ is an endomorphism, then the trace of $f$ is the endomorphism of the unit $\mathbf{1}$ given by the composition

$$
\mathbf{1} \stackrel{\text { coev }}{\longrightarrow} A \otimes A^{\vee} \stackrel{f \otimes \mathrm{id}}{\longrightarrow} A \otimes A^{\vee} \stackrel{\tau}{\simeq} A^{\vee} \otimes A \stackrel{\mathrm{ev}}{\longrightarrow} \mathbf{1}
$$

Throughout this section we fix a smooth and proper morphism $p: X \rightarrow B$. Recall that the projector

$$
P r_{!}^{*}: p_{!}\left(E \wedge p^{*} F\right) \rightarrow p_{!} E \wedge F
$$

is always an isomorphism. In particular, for $E=p^{*} \mathbf{1}_{B}$, we obtain a natural isomorphism

$$
p ! p^{*} \simeq p ! p^{*} \mathbf{1}_{B} \wedge(-)
$$

The projectors

$$
p_{*} p^{!} E \wedge F \stackrel{P r_{*}^{*}}{\longrightarrow} p_{*}\left(p^{!} E \wedge p^{*} F\right) \stackrel{P r^{* !}}{\longrightarrow} p_{*} p^{!}(E \wedge F)
$$

are also isomorphisms, the first because $p$ is proper and the second because $p$ is smooth. For $E=\mathbf{1}_{B}$ we obtain an isomorphism

$$
p_{*} p^{!} \simeq p_{*} p^{!} \mathbf{1}_{B} \wedge(-)
$$

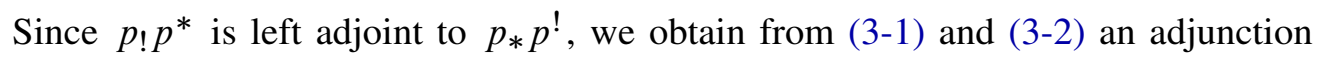
between $p_{!} p^{*} \mathbf{1}_{B} \wedge(-)$ and $p_{*} p^{!} \mathbf{1}_{B} \wedge(-)$, ie, a strong duality between $p ! p^{*} \mathbf{1}_{B}$ and $p_{*} p^{!} \mathbf{1}_{B} \simeq \Sigma_{+}^{\infty} X$. Under the isomorphisms (3-1) and (3-2), the coevaluation map $\mathbf{1}_{B} \rightarrow p_{*} p^{!} \mathbf{1}_{B} \wedge p ! p^{*} \mathbf{1}_{B}$ is the composition of the units

$$
\mathbf{1}_{B} \stackrel{\eta}{\rightarrow} p_{*} p^{*} \mathbf{1}_{B} \stackrel{\eta}{\rightarrow} p_{*} p^{!} p ! p^{*} \mathbf{1}_{B}
$$

and the evaluation map $p_{!} p^{*} \mathbf{1}_{B} \wedge p_{*} p^{!} \mathbf{1}_{B} \rightarrow \mathbf{1}_{B}$ is the composition of the counits

$$
p_{!} p^{*} p_{*} p^{!} \mathbf{1}_{B} \stackrel{\epsilon}{\rightarrow} p_{!} p^{!} \mathbf{1}_{B} \stackrel{\epsilon}{\rightarrow} \mathbf{1}_{B}
$$


Remark 3.5 Composing the coevaluation with the symmetry and the first half of (3-4), we obtain a map $\mathbf{1}_{B} \rightarrow p_{!} p^{!} \mathbf{1}_{B} \simeq \Sigma_{+}^{\infty} X$ in $\mathrm{SH}(B)$. This is the motivic analog of the Becker-Gottlieb transfer in stable parametrized homotopy theory. It is easy to see that integration against the Euler characteristic is equivalent to precomposition with this transfer.

Consider the cartesian square

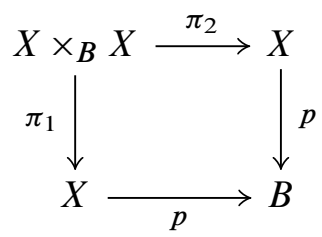

and denote by $\delta: X \hookrightarrow X \times_{B} X$ the diagonal immersion. The key result which will be the basis for the proof of the main theorem in Section 4 is the following description of the trace of an endomorphism:

Proposition 3.6 Let $f: X \rightarrow X$ be a $B$-morphism and let $\omega \in \operatorname{End}\left(\mathbf{1}_{X}\right)$. Then $\operatorname{tr}\left(p_{\sharp} \omega \circ \Sigma_{+}^{\infty} f\right): \mathbf{1}_{B} \rightarrow \mathbf{1}_{B}$ is given by the following composition evaluated at $\mathbf{1}_{B}$ :

$$
\begin{aligned}
& \text { id } \stackrel{\eta}{\rightarrow} p_{*} p^{*} \stackrel{\simeq}{\rightarrow} p_{*} \pi_{1 !} \delta ! \delta^{!} \pi_{2}^{!} p^{*} \stackrel{\epsilon}{\rightarrow} p_{*} \pi_{1 !} \pi_{2}^{!} p^{*} \\
& E x_{! *} \uparrow E x^{* !} \\
& \bigodot p_{!} \pi_{2 *} \pi_{1}^{*} p^{!} \stackrel{\eta}{\rightarrow} p_{!} \pi_{2 *} \delta_{*} \delta^{*} \pi_{1}^{*} p^{!} \stackrel{\widetilde{\sim}}{\rightarrow} p_{!} p^{!} \stackrel{\epsilon}{\rightarrow} \mathrm{id}
\end{aligned}
$$

Here the first loop is

$\left.p_{*} \pi_{1 !} \pi_{2}^{!} p^{*} \stackrel{c}{\simeq} p_{*} f_{!} \pi_{1 !}(f \times \mathrm{id})\right)^{!} \pi_{2}^{!} p^{*} \stackrel{c}{\simeq} p_{*} \pi_{1 !}(f \times \mathrm{id}) !(f \times \mathrm{id}) ! \pi_{2}^{!} p^{*} \stackrel{\epsilon}{\rightarrow} p_{*} \pi_{1 !} \pi_{2}^{!} p^{*}$ and the second loop is

$$
p^{!} \simeq \mathbf{1}_{X} \wedge p^{!}(-) \stackrel{\omega \wedge \text { id }}{\longrightarrow} \mathbf{1}_{X} \wedge p^{!}(-) \simeq p^{!} .
$$

Proof By the base change theorem, the exchange transformations

$$
E x_{!}^{!}: \pi_{1 !} \pi_{2}^{!} \rightarrow p^{!} p_{!} \quad \text { and } E x_{*}^{*}: p^{*} p_{*} \rightarrow \pi_{2 *} \pi_{1}^{*}
$$

are invertible. Lemma B.1 shows that, under these isomorphisms, the first row of the given composition is the coevaluation (3-3) and the second row is the evaluation (3-4). Lemma B.2 shows that the vertical arrow is inverse to the symmetry 
$p_{*} p^{!} \mathbf{1}_{B} \wedge p_{!} p^{*} \mathbf{1}_{B} \simeq p_{!} p^{*} \mathbf{1}_{B} \wedge p_{*} p^{!} \mathbf{1}_{B}$. It remains to prove that, under the isomorphism $\Sigma_{+}^{\infty} X \simeq p_{*} p^{!} \mathbf{1}_{B}$, the first loop corresponds to $\Sigma_{+}^{\infty} f \wedge \mathrm{id}$ and the second loop corresponds to id $\wedge p_{\sharp} \omega$.

Recall from Section 2 that $\Sigma_{+}^{\infty} f$ is the following composition evaluated at $\mathbf{1}_{B}$ :

$$
p_{*} p^{!} \stackrel{c}{\simeq} p_{*} f_{!} f^{!} p^{!} \stackrel{\epsilon}{\rightarrow} p_{*} p^{!}
$$

Under the projection isomorphism (3-2), $\Sigma_{+}^{\infty} f \wedge \mathrm{id}$ is therefore the composition

$$
p_{*} p^{!} p_{!} p^{*} \simeq p_{*} f_{!} f^{!} p^{!} p_{!} p^{*} \stackrel{\epsilon}{\rightarrow} p_{*} p^{!} p_{!} p^{*}
$$

Applying Lemma B.3 to the pair of cartesian squares

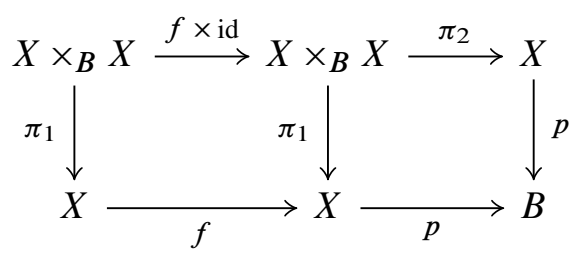

we deduce that (3-7) becomes the first loop under the exchange isomorphisms $E x_{!}^{!}$.

Denote also by $\omega$ the image of $\omega$ under the obvious map $\operatorname{End}\left(\mathbf{1}_{X}\right) \rightarrow \operatorname{End}\left(\operatorname{id}_{\mathrm{SH}(X)}\right)$, so that the second loop is the natural transformation $p_{!} \pi_{2 *} \pi_{1}^{*} \omega p^{!}$. By the compatibility of Thom transformations with the monoidal structure, the transformation $\omega$ commutes with any Thom transformation. The square

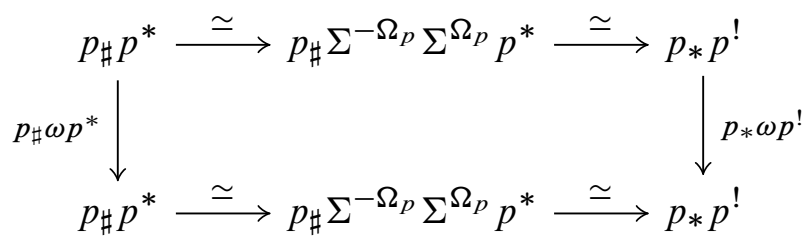

is therefore commutative. Under the natural isomorphism (3-1), id $\wedge p_{\sharp} \omega$ then becomes $p_{!} p^{*} p_{*} \omega p^{!}$, which is the given loop modulo the exchange isomorphism $E x_{*}^{*}$.

In the rest of this section we will give a more explicit description of this duality in a special case which will be used in Section 5. In what follows we often omit the stabilization functor $\Sigma^{\infty}$ from the notation and implicitly view pointed presheaves on $\mathrm{Sm}_{B}$ as objects of $\mathrm{SH}(B)$ (we do not mean to say that the maps we consider are defined unstably, although this will sometimes be the case). 
Definition 3.8 A Euclidean embedding of $X$ is a triple $(s, V, \beta)$ where:

- $s$ is a closed immersion $X \hookrightarrow E$ in $\mathrm{Sm}_{B}$.

- $V$ is a vector bundle on $B$.

- $\beta$ is a path from $s^{*}\left(\mathbb{V}\left(\Omega_{E / B}\right)\right)$ to $p^{*}(V)$ in the $K$-theory groupoid $K(X)$.

The proof of [28, Lemma 2.8] shows that $X$ admits a Euclidean embedding if it is a closed subscheme of a projective bundle over $B$. Note also that, if $X$ admits a Euclidean embedding $(s, V, \beta)$, then it has one where the closed immersion is the zero section of a vector bundle, namely $X \hookrightarrow \mathbb{V}\left(\mathcal{N}_{S}\right)$. In addition to being smooth and proper, we now assume that $X$ admits a Euclidean embedding $(s, V, \beta)$, which we fix once and for all. The path $\beta$ in $K(X)$ determines an isomorphism

$$
\Sigma^{s^{*}\left(\Omega_{E}\right)} \simeq \Sigma^{p^{*}(V)}
$$

of self-equivalences of $\mathrm{SH}(X)$. The short exact sequence

$$
0 \rightarrow \mathcal{N}_{s} \rightarrow s^{*}\left(\Omega_{E}\right) \stackrel{d s}{\longrightarrow} \Omega_{X} \rightarrow 0
$$

of locally free sheaves on $X$ then induces an isomorphism

$$
\Sigma^{-\Omega_{X}} \simeq \Sigma^{-p^{*}(V)} \Sigma^{\mathcal{N}_{s}}
$$

whence

$$
p_{!} \simeq p_{\sharp} \Sigma^{-\Omega_{X}} \simeq p_{\sharp} \Sigma^{-p^{*}(V)} \Sigma^{\mathcal{N}_{S}} \simeq \Sigma^{-V} p_{\sharp} \Sigma^{\mathcal{N}_{s}} .
$$

Finally, by the purity isomorphism, we obtain

$$
p ! \mathbf{1}_{X} \simeq \Sigma^{-V} \frac{E}{E-X} .
$$

It is worth emphasizing that the isomorphism (3-9) depends not only on $s$ and $V$ but also on $\beta$.

Under the isomorphism (3-9), the coevaluation map (3-3) is the $V$-desuspension of a composition

$$
S^{V} \longrightarrow \frac{E}{E-X} \longrightarrow X_{+} \wedge \frac{E}{E-X},
$$

and the evaluation map (3-4) is the $V$-desuspension of a composition

$$
\frac{E}{E-X} \wedge X_{+} \longrightarrow \Sigma^{V} X_{+} \longrightarrow S^{V}
$$

We would like to describe these four maps more explicitly.

Let $\hat{p}: E \rightarrow B$ be the structure map of $E$ and define $\hat{\pi}_{1}$ and $\hat{\pi}_{2}$ by the cartesian square: 


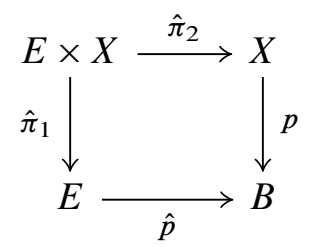

Let also $t: X \hookrightarrow E \times X$ be the composition $(s \times$ id $) \circ \delta$. We will define an isomorphism of short exact sequences:

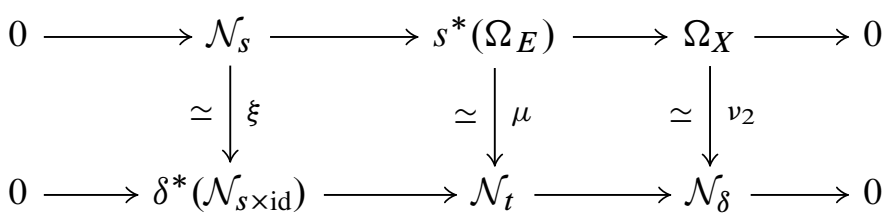

The isomorphism $\xi$ is the composition

$$
\mathcal{N}_{s} \simeq \delta^{*} \pi_{1}^{*}\left(\mathcal{N}_{s}\right) \simeq \delta^{*}\left(\mathcal{N}_{s \times \text { id }}\right)
$$

The isomorphism $v_{2}: \Omega_{X} \stackrel{\sim}{\rightarrow} \mathcal{N}_{\delta}$ is defined so that the composition

$$
\Omega_{X} \stackrel{\nu_{2}}{\rightarrow} \mathcal{N}_{\delta} \hookrightarrow \delta^{*}\left(\Omega_{X \times X}\right)
$$

is $\delta^{*}\left(d \pi_{1}\right)-\delta^{*}\left(d \pi_{2}\right)$. In other words, $\nu_{2}$ is the composition of the canonical isomorphisms

$$
\Omega_{p} \simeq \delta^{*} \pi_{1}^{*}\left(\Omega_{p}\right) \simeq \delta^{*}\left(\Omega_{\pi_{2}}\right) \simeq \mathcal{N}_{\delta} .
$$

It is then clear that the composite equivalence

$$
\mathrm{id} \simeq \delta^{!} \pi_{2}^{!} \simeq \delta^{!} \Sigma^{\Omega_{\pi_{2}}} \pi_{2}^{*} \simeq \Sigma^{\delta^{*}\left(\Omega_{\pi_{2}}\right)} \delta^{!} \pi_{2}^{*} \simeq \Sigma^{\Omega_{p}} \delta^{!} \pi_{2}^{*} \underset{\simeq}{\simeq} \Sigma^{\Omega_{p}-\mathcal{N}_{\delta}} \delta^{*} \pi_{2}^{*} \simeq \Sigma^{\Omega_{p}-\mathcal{N}_{\delta}}
$$

is induced by $\nu_{2}$. Finally, the isomorphism $\mu$ is defined so that the composition

$$
s^{*}\left(\Omega_{E}\right) \stackrel{\mu}{\rightarrow} \mathcal{N}_{t} \hookrightarrow t^{*}\left(\Omega_{E \times X}\right)
$$

is $t^{*}\left(d \hat{\pi}_{1}\right)-t^{*}\left(d\left(s \hat{\pi}_{2}\right)\right)$. It is easy to check that the diagram (3-10) commutes.

Proposition 3.11 Let $(s: X \hookrightarrow E, V, \beta)$ be a Euclidean embedding giving rise to the isomorphism (3-9).

(1) Suppose that $s: X \hookrightarrow E$ is the zero section of a vector bundle $r: E \rightarrow X$. Then the map $p_{*} p^{*} \mathbf{1}_{B} \stackrel{\eta}{\rightarrow} p_{*} p^{!} p_{!} p^{*} \mathbf{1}_{B} \simeq p_{*} p^{!} \mathbf{1}_{B} \wedge p_{!} p^{*} \mathbf{1}_{B}$ is the $V$-desuspension of the composition

$$
\frac{E}{E-X} \stackrel{(r, \text { id })}{\longrightarrow} \frac{X \times E}{X \times(E-X)} \simeq X_{+} \wedge \frac{E}{E-X} .
$$


(2) The map $p_{!} p^{*} \mathbf{1}_{B} \wedge p_{*} p^{!} \mathbf{1}_{B} \simeq p_{!} p^{*} p_{*} p^{!} \mathbf{1}_{B} \stackrel{\epsilon}{\rightarrow} p_{!} p^{!} \mathbf{1}_{B}$ is the $V$-desuspension of the composition

$\frac{E}{E-X} \wedge X_{+} \simeq \frac{E \times X}{(E-X) \times X} \rightarrow \frac{E \times X}{(E \times X)-\Delta_{X}} \stackrel{\Pi}{\simeq} \mathrm{Th}_{X}\left(\mathbb{V}\left(\mathcal{N}_{t}\right)\right) \simeq \Sigma^{V} X_{+}$,

where the last isomorphism is induced by $\mu: \mathcal{N}_{t} \simeq s^{*}\left(\Omega_{E}\right)$ and by $\beta$.

Proof (1) We must check that the two outer compositions in the following diagram coincide:

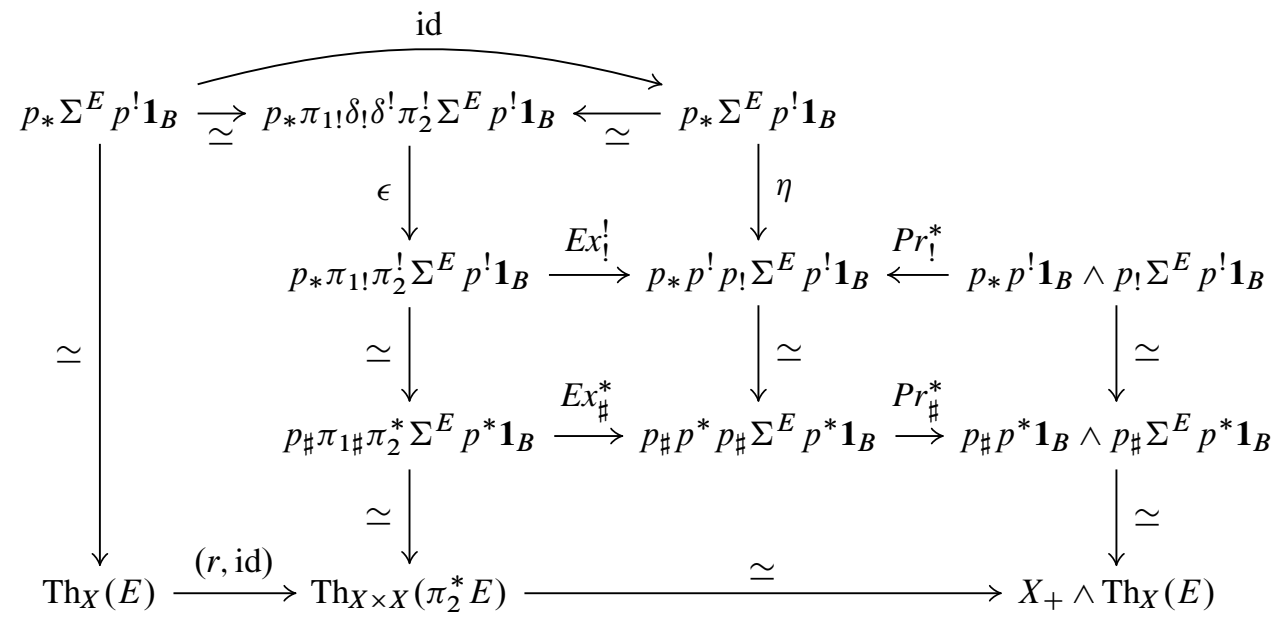

The three vertical isomorphisms in the second row are obtained by getting rid of shrieks and rearranging the resulting Thom transformations. Note that $(r, \mathrm{id}): \mathrm{Th}_{X}(E) \rightarrow$ $\mathrm{Th}_{X \times X}\left(\pi_{2}^{*} E\right)$ is the map induced by the diagonal $\delta: X \rightarrow X \times_{B} X$ and the canonical isomorphism $\delta^{*} \pi_{2}^{*} E \simeq E$. We saw in Section 2 that the left-hand rectangle is commutative. The commutativity of the top square is Lemma B.1(2). Finally, one verifies easily that the lower rectangle is the stabilization of a commutative rectangle of presheaves of pointed sets on $\mathrm{Sm}_{B}$. Thus, the whole diagram is commutative.

(2) We first express the given composition in terms of the six operations. We have

$$
\frac{E \times X}{(E-X) \times X} \simeq p_{\sharp} \hat{\pi}_{2 \sharp}(s \times \mathrm{id})_{*} \pi_{1}^{*} p^{*} \mathbf{1}_{B} \quad \text { and } \quad \frac{E \times X}{(E \times X)-\Delta_{X}} \simeq p_{\sharp} \hat{\pi}_{2 \sharp} t_{*} p^{*} \mathbf{1}_{B},
$$

and the map

$$
\frac{E \times X}{(E-X) \times X} \rightarrow \frac{E \times X}{(E \times X)-\Delta_{X}}
$$

collapsing the complement of the diagonal is given by

$$
p_{\sharp} \hat{\pi}_{2 \sharp}(s \times \mathrm{id})_{*} \pi_{1}^{*} p^{*} \mathbf{1}_{B} \stackrel{\eta}{\rightarrow} p_{\sharp} \hat{\pi}_{2 \sharp}(s \times \mathrm{id})_{*} \delta_{*} \delta^{*} \pi_{1}^{*} p^{*} \mathbf{1}_{B} \simeq p_{\sharp} \hat{\pi}_{2 \sharp} t_{*} p^{*} \mathbf{1}_{B},
$$


as one can see at the level of pointed presheaves on $\mathrm{Sm}_{B}$. Consider the following diagram:

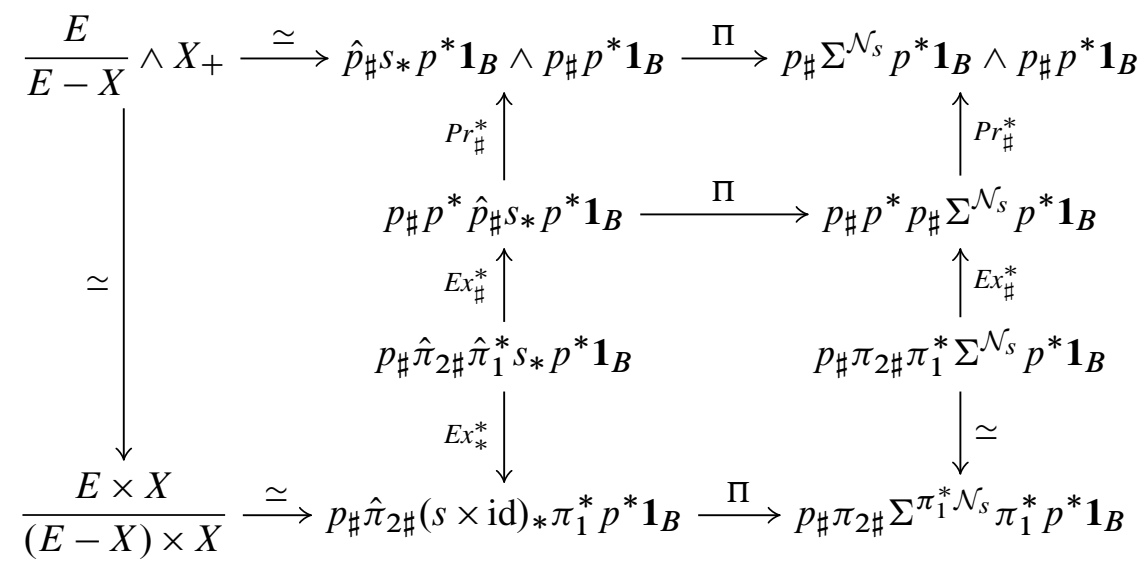

The left rectangle is seen to be commutative at the level of pointed presheaves and the top right square commutes by naturality of $P r_{\sharp}^{*}$. The lower right rectangle becomes an instance of the compatibility of $E x_{*}^{*}$ with compositions of cartesian squares after replacing lower sharps by lower stars. It remains to prove that the composition

$$
\begin{aligned}
p_{\sharp} p^{*} p_{*} \Sigma^{s^{*}\left(\Omega_{E}\right)} p^{*} \simeq p_{\sharp} p^{*} p_{\sharp} \Sigma^{\mathcal{N}_{s}} p^{*} & \stackrel{(3-13)}{\longrightarrow} p_{\sharp} \hat{\pi}_{2 \sharp}(s \times \mathrm{id})_{*} \pi_{1}^{*} p^{*} \\
& \stackrel{(3-12)}{\longrightarrow} p_{\sharp} \hat{\pi}_{2 \sharp} t_{*} p^{*} \stackrel{\Pi}{\simeq} p_{\sharp} \Sigma^{\mathcal{N}_{t}} p^{*} \stackrel{\mu}{\simeq} p_{\sharp} \Sigma^{s^{*}\left(\Omega_{E}\right)} p^{*}
\end{aligned}
$$

is equal to the counit $\epsilon$ (when evaluated at $\mathbf{1}_{B}$ ). One finds these two maps as the boundary of the following diagram, after applying $p_{\sharp}(-) p^{*}$ :

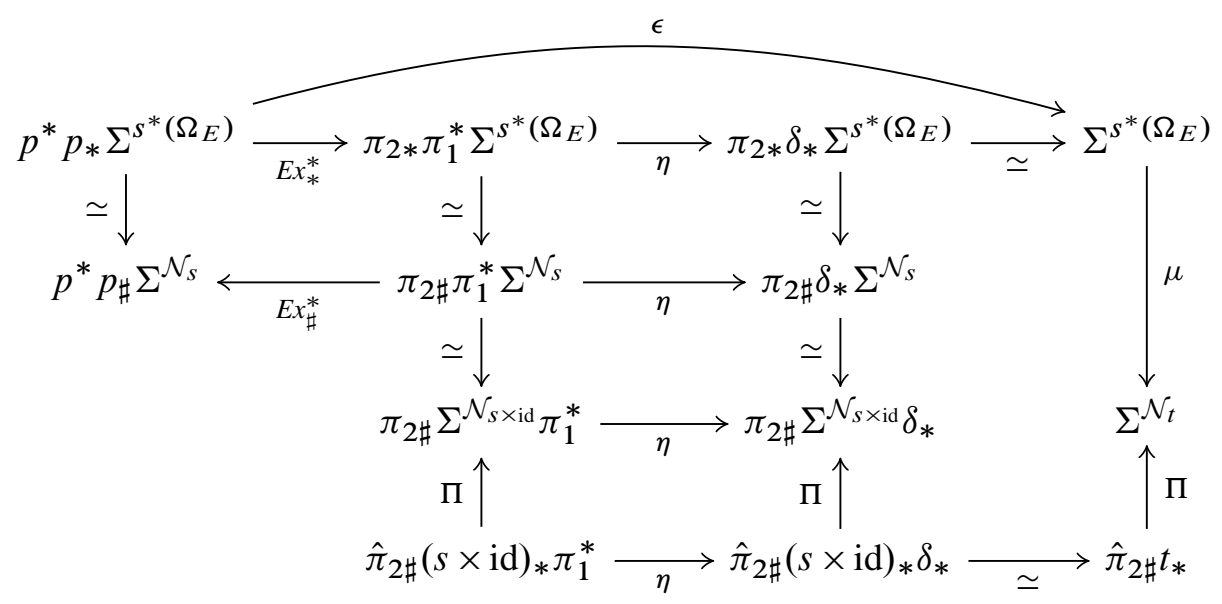


We claim that this diagram commutes. The topmost face commutes by Lemma B.1(1) and the commutativity of the four small squares is clear. The large rectangle may be decomposed as follows:

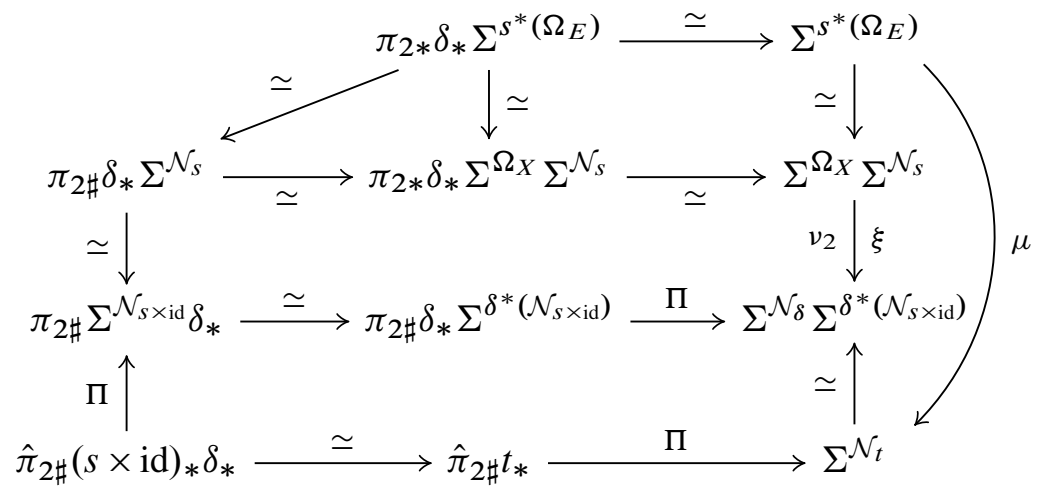

The rightmost face commutes by (3-10) and the middle rectangle commutes by the definitions of $\xi$ and $\nu_{2}$. Finally, the bottom rectangle commutes by the compatibility of the purity isomorphisms with the composition of the closed immersions $\delta$ and $s \times$ id [3, Section 1.6.4].

The counit $\epsilon: p ! p^{!} \mathbf{1}_{B} \rightarrow \mathbf{1}_{B}$ is of course the map $\Sigma_{+}^{\infty} p: \Sigma_{+}^{\infty} X \rightarrow \Sigma_{+}^{\infty} B=\mathbf{1}_{B}$. The unit $\eta: \mathbf{1}_{B} \rightarrow p_{*} p^{*} \mathbf{1}_{B}$ is more difficult to describe explicitly and we do not know how to do it in any kind of generality. ${ }^{5}$ However, we can at least give a useful characterization of $\eta$ :

Proposition 3.14 Let $(s: X \hookrightarrow E, V, \beta)$ be a Euclidean embedding and let

$$
\zeta: S^{V} \rightarrow \frac{E}{E-X}
$$

be a map in $\mathrm{SH}(B)$. The following conditions are equivalent:

(1) Under the isomorphism (3-9), $\zeta$ is the $V$-suspension of the unit $\eta: \mathbf{1}_{B} \rightarrow$ $p_{*} p^{*} \mathbf{1}_{B}$.

(2) The composition

$$
\Sigma^{V} X_{+} \stackrel{\zeta \wedge \text { id }}{\rightarrow} \frac{E}{E-X} \wedge X_{+} \rightarrow \Sigma^{V} X_{+} \stackrel{p}{\rightarrow} S^{V},
$$

where the second map is given in Proposition 3.11(2), is equal to $\Sigma^{V} \Sigma_{+}^{\infty} p$.

${ }^{5}$ If $X$ is a closed subscheme of a projective bundle over $B$, it is possible that the unstable map $S^{V} \rightarrow E /(E-X)$ constructed by Voevodsky in [28, Theorem 2.11(2)] (for a specific Euclidean embedding $(s, V, \beta))$ stabilizes to $\eta$, but we did not check it. 
Proof Since the unit $\eta: \mathbf{1}_{B} \rightarrow p_{*} p^{*} \mathbf{1}_{B}$ is dual to $\epsilon: p_{!} p^{!} \mathbf{1}_{B} \rightarrow \mathbf{1}_{B}$, it is determined by the equation ev $\circ(\eta \wedge \mathrm{id})=\epsilon$. The equivalence of (1) and (2) is now clear by Proposition 3.11(2).

In Section 5, we will define a map $\zeta$ satisfying the condition of Proposition 3.14(2) when $B$ is a field and $X$ is a finite separable extension of $B$. As a result, the duality will be completely explicit in this case.

\section{Proof of the main theorem}

We prove Theorem 1.3. As a warm-up, assume that $X$ admits a Euclidean embedding $(s, V, \beta)$, chosen such that $s$ is the zero section of a vector bundle $r: E \rightarrow X$. By Proposition 3.11, the trace of $\Sigma_{+}^{\infty} f$ is then the $V$-desuspension of a composition

$$
\begin{aligned}
S^{V} \rightarrow \frac{E}{E-X} \stackrel{(r, \text { id })}{\longrightarrow} & \frac{X \times E}{X \times(E-X)} \\
\stackrel{f \times \text { id }}{\longrightarrow} & \frac{X \times E}{X \times(E-X)} \\
& \stackrel{\tau}{\simeq} \frac{E \times X}{(E-X) \times X} \\
& \rightarrow \frac{E \times X}{(E \times X)-\Delta_{X}} \simeq \Sigma^{V} X_{+} \rightarrow S^{V},
\end{aligned}
$$

where $\tau$ is the monoidal symmetry. Ignoring the first and last arrows, it is clear that the remaining composition factors through $E /\left(E-X^{f}\right)$ and hence that $\operatorname{tr}\left(\Sigma_{+}^{\infty} f\right)=0$ if $X^{f}$ is empty (compare this argument with the proof of the Lefschetz-Hopf theorem by Dold and Puppe in [8]). It is possible to prove the more precise statement of Theorem 1.3 in this explicit setting, but, to treat the general case where $X$ is proper over an arbitrary base, we will now switch to the formalism of six operations. Throughout this section we use the following notation:
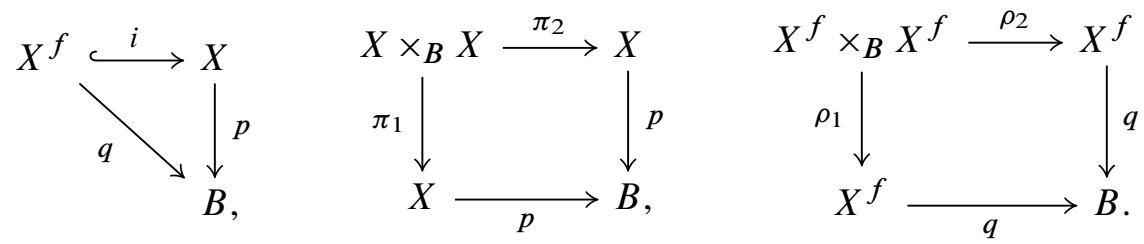

Let also $\delta: X \hookrightarrow X \times_{B} X$ and $\theta: X^{f} \hookrightarrow X^{f} \times_{B} X^{f}$ be the diagonal maps and let $\gamma=(f \times$ id $) \circ \delta$ be the graph of $f$. For the moment we do not assume that $f$ has regular fixed points. 
Recall from Proposition 3.6 that $\operatorname{tr}\left(\Sigma_{+}^{\infty} f\right)$ can be expressed as a certain composition

$$
\mathrm{id} \stackrel{\eta}{\rightarrow} p_{*} p^{*} \rightarrow p_{!} p^{!} \stackrel{\epsilon}{\rightarrow} \mathrm{id}
$$

evaluated at $\mathbf{1}_{B}$, where the transformation $p_{*} p^{*} \rightarrow p_{!} p^{!}$is the solid boundary of the following commutative diagram:

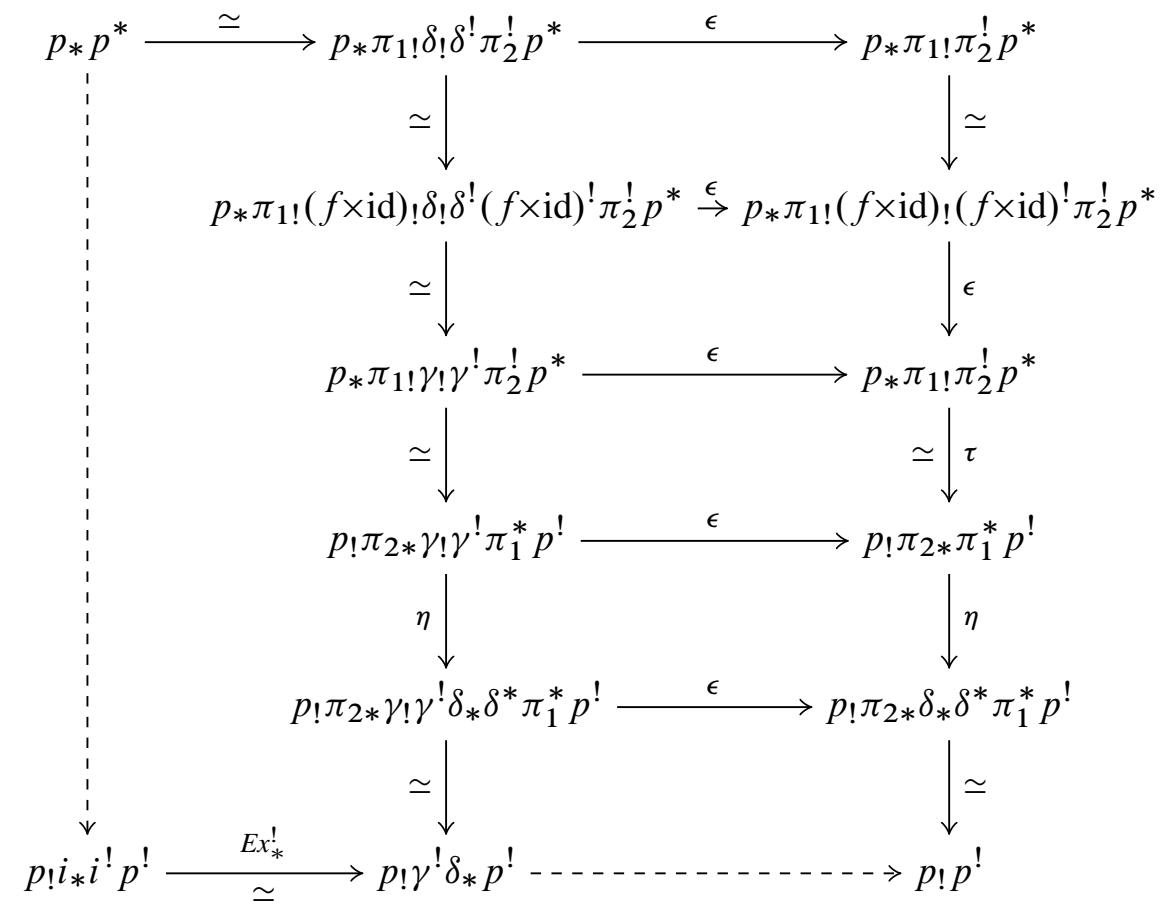

The map at the bottom left is the exchange transformation $E x_{*}^{!}: i_{*} i^{!} \rightarrow \gamma^{!} \delta_{*}$ associated with the cartesian square

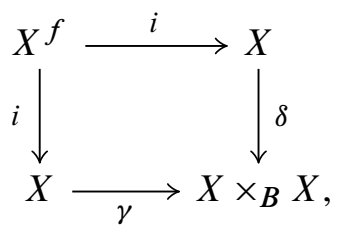

and it is an isomorphism by the base change theorem. The dashed arrows in (4-1) can then be defined so as to make the diagram commute. Lemma B.4 shows that the bottom row in (4-1) is the counit $\epsilon: p_{!} i_{*} i^{!} p^{!} \rightarrow p_{!} p^{!}$. Note that this diagram already proves Corollary 1.4: if $X^{f}$ is empty, then $i_{*} i !$ is the zero functor and hence $\operatorname{tr}\left(\Sigma_{+}^{\infty} f\right)=0$. 
The dashed arrow $p_{*} p^{*} \rightarrow p_{!} i_{*} i^{!} p^{!}$in (4-1) is the composition of the right column and the bottom row in the following diagram:

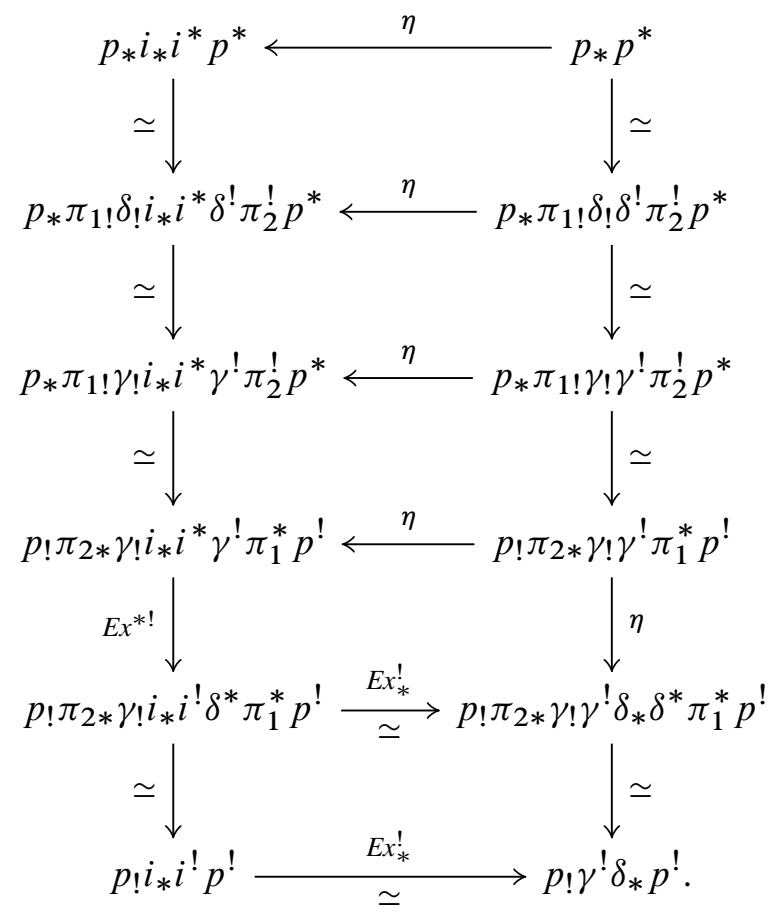

The commutativity of each square in this diagram is clear, except that of the fourth square which follows from the definition of the exchange transformation $E x_{*}^{!}$in terms of $E x^{* !}$ (see [3, Section 1.2.4]).

The left column of (4-3) is a natural transformation $q_{*} q^{*} \rightarrow q ! q^{!}$which, by (4-1) and Lemma B.4, makes the following diagram commute:

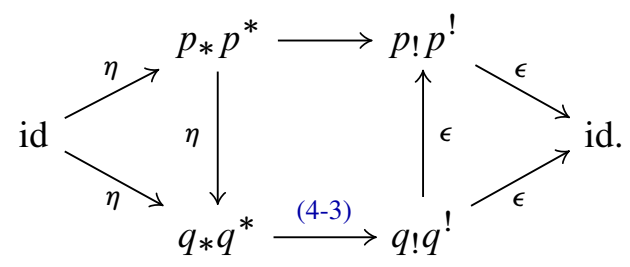

Assume now that $f$ has regular fixed points, ie that $q$ is smooth and that $\mathrm{id}-i^{*}(d f)$ restricts to an automorphism $\phi: \mathcal{N}_{i} \stackrel{\sim}{\rightarrow} \mathcal{N}_{i}$. By Proposition 3.6, $\int_{X^{f}}\langle\phi\rangle d \chi$ is a certain composition

$$
\mathrm{id} \stackrel{\eta}{\rightarrow} q_{*} q^{*} \rightarrow q ! q^{!} \stackrel{\epsilon}{\rightarrow} \mathrm{id}
$$


evaluated at $\mathbf{1}_{B}$. In view of (4-4), to conclude the proof of Theorem 1.3, it will suffice to show that the segment $q_{*} q^{*} \rightarrow q ! q^{!}$in (4-5) is equal to the left column of (4-3). This segment (as given by Proposition 3.6) is the composition of the top row and the right vertical arrows in the following diagram:

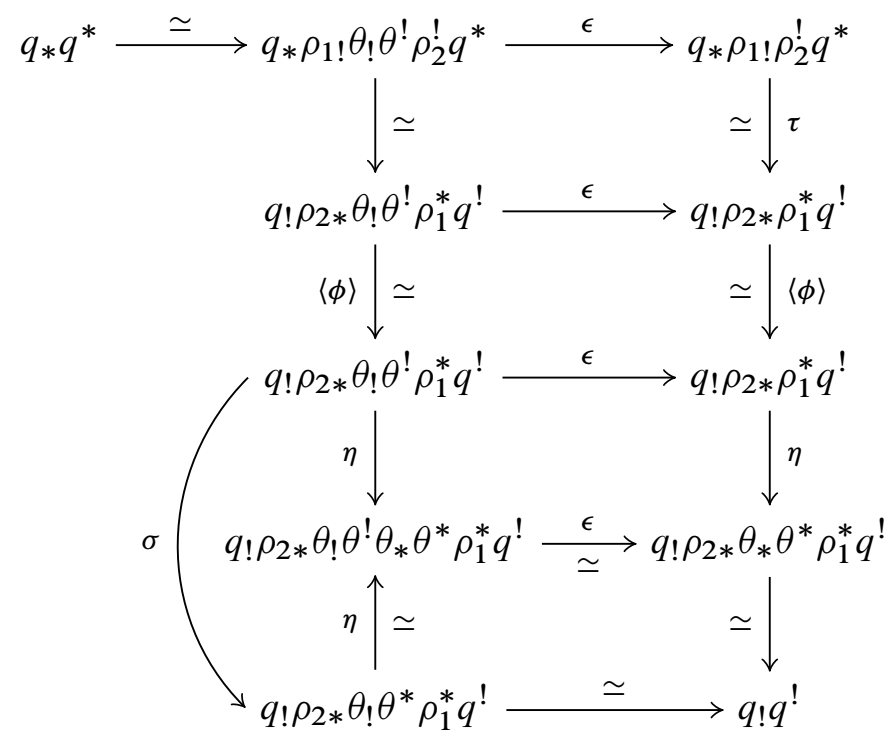

where $\langle\phi\rangle$ acts after $q^{!}$in both columns. Each square commutes by the naturality of the given transformations, except the last square which commutes by a triangle identity for the adjunction $\theta_{*} \simeq \theta_{!} \dashv \theta^{!}$. The triangle at the bottom left commutes by Lemma B.5.

Remark 4.7 In the diagram (4-6), one can replace $\langle\phi\rangle$ by any endomorphism of $\mathbf{1}_{X^{f}}$ and $X^{f}$ itself by any smooth proper $B$-scheme. Theorem 1.5 follows from the observation that the natural transformation

$$
\sigma: \theta^{!} \rho_{1}^{*} \rightarrow \theta^{*} \rho_{1}^{*}
$$

is zero if $\left[\Omega_{X^{f}}\right]=\left[\mathcal{O}_{X^{f}}\right]+[\mathcal{E}]$ in $K_{0}\left(X^{f}\right)$. Indeed, by Proposition A.4, this transformation can be identified to the transformation $\Sigma^{-\mathcal{N}_{\theta}} \rightarrow \Sigma^{0}$ induced by the epimorphism $\mathcal{N}_{\theta} \rightarrow 0$. Since $\mathcal{N}_{\theta}$ is isomorphic to $\Omega_{X^{f}}$, this transformation factors through the transformation $\Sigma^{-\mathbb{A}^{1}} \rightarrow \Sigma^{0}$ induced by the zero section of the trivial line bundle, which is clearly zero (see for example [3, Lemme 1.6.1]).

We now compare the left column of (4-3) with the left column of (4-6). Both columns are of the form $q_{*}\left(q^{*} \rightarrow q^{!}\right)$, where the respective maps $q^{*} \rightarrow q^{!}$are the left and right columns of the following diagram: 


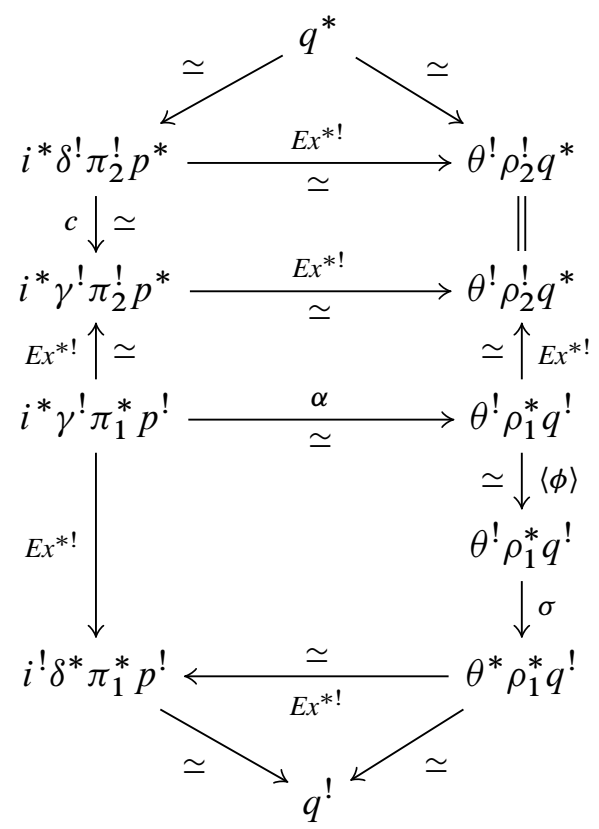

Here the isomorphism $\alpha$ is defined by the commutativity of the second square. The commutativity of the first square is clear. Theorem 1.3 is thus reduced to the commutativity of the pentagon

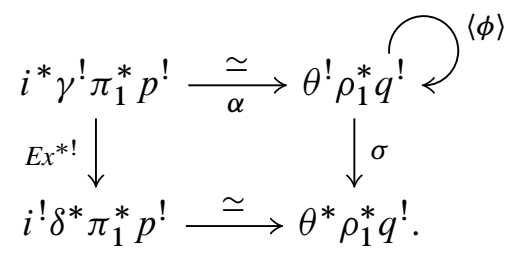

This is the heart of the proof. By transforming the stars into shrieks, this pentagon becomes

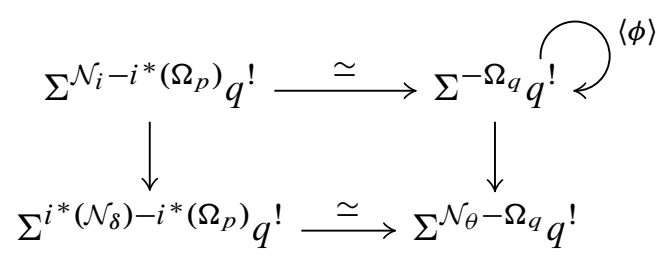

and we now identify the four unlabeled arrows. By definition of $\alpha$, the top map in (4-8) is induced by the short exact sequence

$$
0 \rightarrow \mathcal{N}_{i} \rightarrow i^{*}\left(\Omega_{p}\right) \stackrel{d i}{\longrightarrow} \Omega_{q} \rightarrow 0 .
$$

Denote by $v_{1}: \Omega_{X} \stackrel{\sim}{\rightarrow} \mathcal{N}_{\delta}$ the isomorphism for which the composition 


$$
\Omega_{X} \stackrel{\nu_{1}}{\rightarrow} \mathcal{N}_{\delta} \hookrightarrow \delta^{*}\left(\Omega_{X \times_{B} X}\right)
$$

is $\delta^{*}\left(d \pi_{2}\right)-\delta^{*}\left(d \pi_{1}\right)$. The composite isomorphism

$$
\mathrm{id} \simeq \delta^{*} \pi_{1}^{*} \simeq \Sigma^{\mathcal{N}_{\delta}-\Omega_{p}} \delta^{!} \pi_{1}^{!} \simeq \Sigma^{\mathcal{N}_{\delta}-\Omega_{p}}
$$

is then induced by $\nu_{1}$, and similarly for the isomorphism id $\simeq \Sigma^{\mathcal{N}_{\theta}-\Omega_{q}}$ (for more details, see the discussion of the isomorphism $v_{2}$ before Proposition 3.11). Under these trivializations, the bottom map in (4-8) is just the identity $q^{!} \rightarrow q^{!}$. The vertical maps in (4-8) can be identified using Proposition A.4. Applying Proposition A.4 to the cartesian square (4-2) shows that the left vertical arrow in (4-8) is $\Sigma^{\psi}$ where $\psi: i^{*}\left(\mathcal{N}_{\delta}\right) \rightarrow \mathcal{N}_{i}$ is the epimorphism induced by (4-2). Explicitly, $\psi$ is determined by the following diagram of short exact sequences:

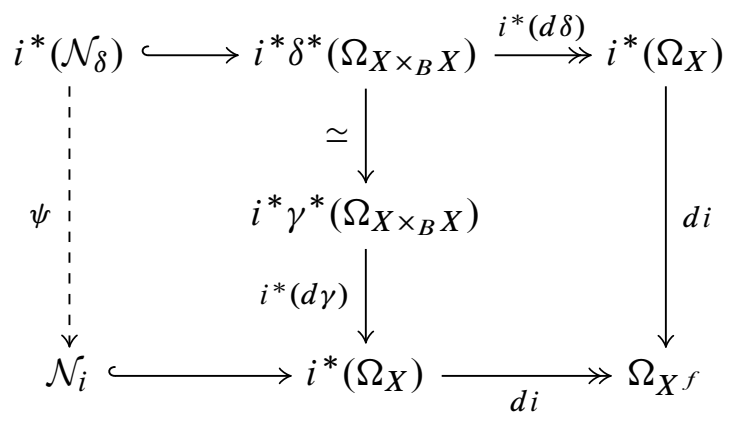

Finally, applying Proposition A.4 to the pullback of $\theta$ along itself shows that the right arrow in (4-8) is $\Sigma^{\zeta}$ where $\zeta$ is the epimorphism $\mathcal{N}_{\theta} \rightarrow 0$. The commutativity of (4-8) is thereby reduced to the commutativity of the following diagram:

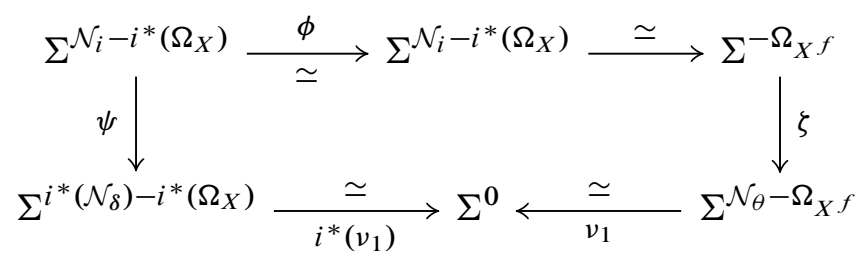

Applying $\Sigma^{i^{*}}\left(\Omega_{X}\right)$, it is equivalent to check that the following diagram commutes:

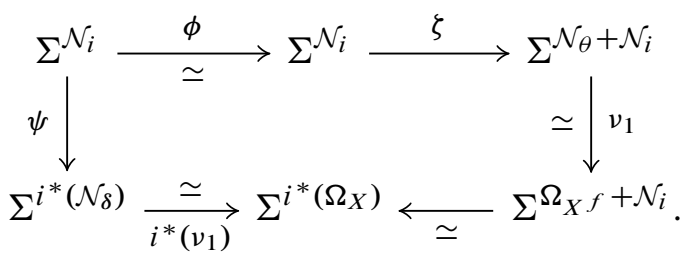


By the naturality of the isomorphisms (2-3), it will suffice to verify the commutativity of the following diagram of locally free sheaves:

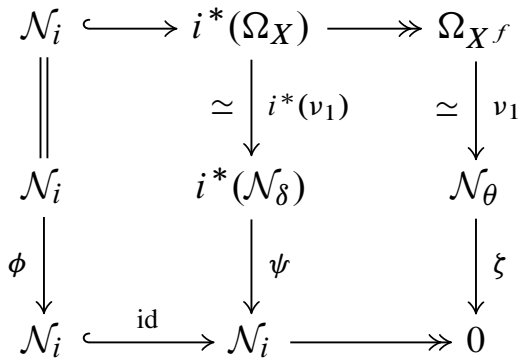

This can be checked on sections as follows. Let $[x]$ be a section of $\mathcal{N}_{i}$, represented by a section $x$ of $\mathcal{O}_{X}$ vanishing on $X^{f}$. Its image in $i^{*}\left(\Omega_{X}\right)$ is $i^{*}(d x)$. By the definitions of $v_{1}$ and $\psi$, we have

$$
\psi\left(i^{*}\left(v_{1}(d x)\right)\right)=\psi\left(i^{*}[1 \otimes x-x \otimes 1]\right)=[x-x \circ f]=\phi([x]),
$$

as desired. This concludes the proof of Theorem 1.3.

\section{The Euler characteristic of separable field extensions}

In this section we prove Theorem 1.9. When $L=k$, the statement of Theorem 1.9 reduces to the following lemma:

Lemma 5.1 Let $V$ be a finite-dimensional vector space over $k$ and let $\phi$ be a linear automorphism of $V$. Then $\langle\phi\rangle=\langle\operatorname{det}(\phi)\rangle$ in $\operatorname{End}\left(\mathbf{1}_{k}\right)$.

Proof Recall from Section 2 that $\langle-\rangle$ factors through a group homomorphism $K_{1}(k) \rightarrow \operatorname{Aut}\left(\mathbf{1}_{k}\right)$. The lemma then follows from the fact that the determinant induces an isomorphism $K_{1}(k) \simeq k^{\times}$.

In view of Lemma 5.1, the following proposition completes the proof of Theorem 1.9:

Proposition 5.2 Let $k \subset L$ be a finite separable field extension. For any $\omega \in$ $\operatorname{End}\left(\mathbf{1}_{L}\right) \simeq \mathrm{GW}(L)$,

$$
\int_{L} \omega d \chi=\operatorname{Tr}_{L / k}(\omega)
$$

Proof Combine Lemmas 5.8, 5.9 and 5.10. 
Recall that, if $p: X \rightarrow B$ is étale, there are canonical isomorphisms $p^{!} \simeq p^{*}$ and $p_{!} \simeq p_{\sharp}$. If moreover $p$ is finite, we therefore have a canonical isomorphism $p_{*} \simeq p_{\sharp}$.

Lemma 5.3 Let $p: X \rightarrow B$ be a finite étale morphism and let $\omega \in \operatorname{End}\left(\mathbf{1}_{X}\right)$. Then $\int_{X} \omega d \chi \in \operatorname{End}\left(\mathbf{1}_{B}\right)$ is the composition

$$
\mathbf{1}_{B} \stackrel{\eta}{\rightarrow} p_{*} \mathbf{1}_{X} \simeq p_{\sharp} \mathbf{1}_{X} \stackrel{p_{\sharp} \omega}{\longrightarrow} p_{\sharp} \mathbf{1}_{X} \stackrel{\epsilon}{\rightarrow} \mathbf{1}_{B} .
$$

Proof By Proposition 3.6, $\int_{X} \omega d \chi$ is the composition

$$
\begin{aligned}
\text { id } \stackrel{\eta}{\rightarrow} p_{*} p^{*} \stackrel{\simeq}{\rightarrow} p_{*} \pi_{1 !} \delta_{!} \delta^{!} \pi_{2}^{!} p^{*} \stackrel{\epsilon}{\rightarrow} p_{*} \pi_{1 !} \pi_{2}^{!} p^{*} \\
\\
\qquad x_{! *} \uparrow E x^{* !} \\
\bigodot p_{!} \pi_{2 *} \pi_{1}^{*} p^{!} \stackrel{\eta}{\rightarrow} p ! \pi_{2 *} \delta_{*} \delta^{*} \pi_{1}^{*} p^{!} \stackrel{\simeq}{\rightarrow} p ! p^{!} \stackrel{\epsilon}{\rightarrow} \mathrm{id},
\end{aligned}
$$

where the loop is $\omega$ acting after $p^{!}$. By naturality, we can move this loop to the next-to-last position $p_{!} p^{!}$. It then remains to prove that the composition $p_{*} p^{*} \rightarrow p_{!} p^{\text {! }}$ (without the loop) is the canonical isomorphism. The morphisms $p, \delta, \pi_{1}$ and $\pi_{2}$ are all finite étale, so we can replace everywhere upper shrieks by upper stars and lower stars by lower shrieks. This operation transforms the exchange isomorphisms $E x_{\text {!* }}$ and $E x^{* !}$ into the connection isomorphisms $E x ! !$ and $E x^{* *}$, and we must then prove that the following composition is the identity:

$$
p_{!} p^{*} \simeq p_{!} \pi_{1 !} \delta_{!} \delta^{*} \pi_{2}^{*} p^{*} \rightarrow p_{!} \pi_{1 !} \pi_{2}^{*} p^{*} \stackrel{c}{\simeq} p_{!} \pi_{2 !} \pi_{1}^{*} p^{*} \rightarrow p_{!} \pi_{2 !} \delta_{!} \delta^{*} \pi_{1}^{*} p^{*} \simeq p ! p^{*} .
$$

Using the coherence of the connection isomorphisms, we are reduced to proving that the composition

$$
\delta_{!} \delta^{*} \simeq \delta_{!} \delta^{!} \stackrel{\epsilon}{\rightarrow} \mathrm{id} \stackrel{\eta}{\rightarrow} \delta_{*} \delta^{*} \simeq \delta_{!} \delta^{*}
$$

is the identity. This is clear since $\delta$ is an open and closed immersion.

Fix a base field $k$. Recall that there is a canonical isomorphism $S^{\mathbb{A}^{1}} \simeq \mathbb{P}_{k}^{1} / \infty$ in $\mathrm{H}_{*}(k)$ given by the zig-zag

$$
\mathbb{A}_{k}^{1} /\left(\mathbb{A}_{k}^{1}-0\right) \rightarrow \mathbb{P}_{k}^{1} /\left(\mathbb{P}_{k}^{1}-0\right) \leftarrow \mathbb{P}_{k}^{1} / \infty
$$

Lemma 5.4 Let $a$ : $\operatorname{Spec} k \hookrightarrow \mathbb{A}_{k}^{1}$ be a rational point. Then the composition

$$
S^{\mathbb{A}^{1}} \simeq \mathbb{P}_{k}^{1} / \infty \rightarrow \frac{\mathbb{P}_{k}^{1}}{\mathbb{P}_{k}^{1}-a} \simeq S^{\mathcal{N}_{a}} \simeq S^{\mathbb{A}^{1}}
$$


is the identity in $\mathrm{H}_{*}(k)$, where the last isomorphism is induced by the trivialization $\mathcal{O}_{k} \simeq \mathcal{N}_{a}, 1 \mapsto t-a$.

Proof Suppose first that $a=0$. We must then show that the composition

$$
\frac{\mathbb{A}_{k}^{1}}{\mathbb{A}_{k}^{1}-0} \rightarrow \frac{\mathbb{P}_{k}^{1}}{\mathbb{P}_{k}^{1}-0} \simeq S^{\mathcal{N}_{0}} \simeq S^{\mathbb{A}^{1}}
$$

is the identity, which follows from [28, Lemma 2.2]. The general case is easily reduced to the case $a=0$ by noting that the map

$$
\mathbb{P}^{1} / \infty \rightarrow \mathbb{P}^{1} / \infty, \quad[x: y] \mapsto[x+a y: y],
$$

is $\mathbb{A}^{1}$-homotopic to the identity.

Lemma 5.5 Let $L$ be a finite separable extension of $k, p$ : Spec $L \rightarrow \operatorname{Spec} k$ the corresponding morphism of schemes and $a$ : Spec $L \hookrightarrow \mathbb{A}_{k}^{1}$ a closed immersion with minimal polynomial $f \in k[t]$. Then the map

$$
\mathbf{1}_{k} \stackrel{\eta}{\rightarrow} p_{*} \mathbf{1}_{L} \simeq p_{\sharp} \mathbf{1}_{L}
$$

is the $\mathbb{A}^{1}$-desuspension of the composition

$$
\Sigma^{\mathbb{A}^{1}}(\operatorname{Spec} k)_{+} \simeq \mathbb{P}_{k}^{1} / \infty \rightarrow \frac{\mathbb{P}_{k}^{1}}{\mathbb{P}_{k}^{1}-a} \simeq \operatorname{Th}_{\operatorname{Spec} L}\left(\mathcal{N}_{a}\right) \simeq \Sigma^{\mathbb{A}^{1}}(\operatorname{Spec} L)_{+},
$$

where $\mathcal{N}_{a}$ is trivialized via $f / f^{\prime}(a)$.

Proof Denote by $\zeta: S^{\mathbb{A}^{1}} \rightarrow \mathbb{P}_{k}^{1} /\left(\mathbb{P}_{k}^{1}-a\right)$ the first part of the given composition. The immersion $a$ : Spec $L \hookrightarrow \mathbb{P}_{k}^{1}$ and the given trivialization $\mathcal{N}_{a} \simeq \mathcal{O}_{L}$ form a Euclidean embedding of $\operatorname{Spec} L$ (Definition 3.8), and the second part of the given composition is the $\mathbb{A}^{1}$-suspension of the isomorphism (3-9) constructed from this Euclidean embedding. By Proposition 3.14, it therefore suffices to show that the composition

$$
\Sigma^{\mathbb{A}^{1}}(\operatorname{Spec} L)_{+} \stackrel{\zeta \wedge \text { id }}{\rightarrow} \frac{\mathbb{P}_{L}^{1}}{\mathbb{P}_{L}^{1}-a_{L}} \stackrel{h}{\rightarrow} \Sigma^{\mathbb{A}^{1}}(\operatorname{Spec} L)_{+} \stackrel{p}{\rightarrow} S^{\mathbb{A}^{1}}
$$

is equal to $\Sigma^{\mathbb{A}^{1}} p_{+}$in $\mathrm{H}_{*}(k)$, where $h$ is the map described in Proposition 3.11(2). Explicitly, $h$ is the composition

$$
\frac{\mathbb{P}_{L}^{1}}{\mathbb{P}_{L}^{1}-a_{L}} \rightarrow \frac{\mathbb{P}_{L}^{1}}{\mathbb{P}_{L}^{1}-\tilde{a}} \simeq \operatorname{Th}_{\operatorname{Spec} L}\left(\mathcal{N}_{\tilde{a}}\right) \simeq \Sigma^{\mathbb{A}^{1}}(\operatorname{Spec} L)_{+},
$$


where:

- $a_{L}: \operatorname{Spec}\left(L \otimes_{k} L\right) \hookrightarrow \mathbb{P}_{L}^{1}$ is the base change of $a$.

- $\tilde{a}=a_{L} \circ \delta$ is the $L$-point of $\mathbb{P}_{L}^{1}$ above $a$.

- $\mathcal{N}_{\tilde{a}}$ is trivialized via the isomorphism

$$
\mathcal{N}_{a} \simeq a^{*}\left(\Omega_{\mathbb{P}_{k}^{1}}\right) \stackrel{\tilde{a}^{*}\left(d \hat{\pi}_{1}\right)}{\longrightarrow} \tilde{a}^{*}\left(\Omega_{\mathbb{P}_{L}^{1}}\right) \simeq \mathcal{N}_{\tilde{a}}
$$

and the given trivialization of $\mathcal{N}_{a}$, where $\hat{\pi}_{1}: \mathbb{P}_{L}^{1} \rightarrow \mathbb{P}_{k}^{1}$ is the base change of $p$.

With the identifications

$$
\mathcal{N}_{a}=(f) \otimes_{k[t]} L \quad \text { and } \quad \mathcal{N}_{\tilde{a}}=(t-a) \otimes_{L[t]} L,
$$

the isomorphism (5-7) is induced by the inclusion $(f) \subset(t-a)$. If $f(t)=(t-a) g(t)$ in $L[t]$, we have

$$
f(t) \otimes \frac{1}{f^{\prime}(a)}=(t-a) g(t) \otimes \frac{1}{f^{\prime}(a)}=(t-a) \otimes \frac{g(a)}{f^{\prime}(a)}=(t-a) \otimes 1 .
$$

Thus, since $\mathcal{N}_{a}$ is trivialized by $f / f^{\prime}(a), \mathcal{N}_{\tilde{a}}$ is trivialized by the monomial $t-a$. The composition

$$
\Sigma^{\mathbb{A}^{1}}(\operatorname{Spec} L)_{+} \stackrel{\zeta \wedge \text { id }}{\longrightarrow} \frac{\mathbb{P}_{L}^{1}}{\mathbb{P}_{L}^{1}-a_{L}} \stackrel{h}{\rightarrow} \Sigma^{\mathbb{A}^{1}}(\operatorname{Spec} L)_{+}
$$

is therefore the identity in $\mathrm{H}_{*}(k)$ by Lemma 5.4 (applied to $\tilde{a}$ : $\operatorname{Spec} L \hookrightarrow \mathbb{A}_{L}^{1}$ ), and hence (5-6) is equal to $\Sigma^{\mathbb{A}^{1}} p_{+}$, as was to be shown.

Let $v$ be a finite place of the field of rational functions $k(t)$ with residue field $\kappa(v)$. As a $k$-vector space, $\kappa(v)$ has a basis $\left\{1, t, \ldots, t^{n-1}\right\}$, where $n=\operatorname{deg}(v)$. We let

$$
\tau_{v}^{\mathrm{Sch}}: \mathrm{GW}(\kappa(v)) \rightarrow \mathrm{GW}(k)
$$

be the Scharlau transfer associated with the $k$-linear map $\kappa(v) \rightarrow k$ defined by

$$
t^{i} \mapsto \begin{cases}0 & \text { if } 0 \leq i \leq n-2, \\ 1 & \text { if } i=n-1 .\end{cases}
$$

Let also

$$
\tau_{v}^{\text {geom }}: \mathrm{GW}(\kappa(v)) \rightarrow \mathrm{GW}(k)
$$

be the geometric transfer defined by Morel in [20, Section 4.2]. 
Lemma 5.8 Let $v$ be a finite separable place of $k(t)$ with minimal polynomial $f \in$ $k[t]$. Then, for any $\omega \in \mathrm{GW}(\kappa(v))$,

$$
\operatorname{Tr}_{\kappa}(v) / k(\omega)=\tau_{v}^{\mathrm{Sch}}\left(\left\langle f^{\prime}(t)\right\rangle \omega\right) .
$$

Proof By [26, III, Section 6, Lemme 2], we have

$$
\operatorname{Tr}_{\kappa(v) / k}\left(\frac{t^{i}}{f^{\prime}(t)}\right)= \begin{cases}0 & \text { if } 0 \leq i \leq n-2, \\ 1 & \text { if } i=n-1\end{cases}
$$

This immediately implies the lemma.

Lemma 5.9 Let $v$ be a finite separable place of $k(t)$ with minimal polynomial $f \in$ $k[t]$. Then, for any $\omega \in \mathrm{GW}(\kappa(v))$,

$$
\int_{\kappa(v)} \omega d \chi=\tau_{v}^{\text {geom }}\left(\left\langle f^{\prime}(t)\right\rangle \omega\right)
$$

Proof If $a$ : $\operatorname{Spec} \kappa(v) \hookrightarrow \mathbb{P}_{k}^{1}$ is the closed immersion corresponding to $v, \tau_{v}^{\text {geom }}$ is the transfer along the same composition as in Lemma 5.5, except that the conormal sheaf $\mathcal{N}_{a}$ is trivialized via $f$ (see [20, Section 4.2]). The lemma thus follows from Lemmas 5.3 and 5.5.

Lemma 5.10 For every finite place $v$ of $k(t), \tau_{v}^{\text {Sch }}=\tau_{v}^{\text {geom }}$.

Proof For each place $v$ of $k(t)$, we choose a uniformizer $\pi_{v} \in \mathcal{O}_{v}$ as follows: if $v$ is finite, let $\pi_{v}$ be its minimal polynomial, and let $\pi_{\infty}=-1 / t$. By [20, Theorem 3.15], there is a unique residue homomorphism

$$
\partial_{v}: K_{*+1}^{\mathrm{MW}}(k(t)) \rightarrow K_{*}^{\mathrm{MW}}(\kappa(v))
$$

commuting with multiplication by the Hopf element $\eta \in K_{-1}^{\mathrm{MW}}$ and such that, if $u_{1}, \ldots, u_{n} \in \mathcal{O}_{v}^{\times}$,

$$
\partial_{v}\left(\left[\pi_{v}\right]\left[u_{1}\right] \cdots\left[u_{n}\right]\right)=\left[\bar{u}_{1}\right] \cdots\left[\bar{u}_{n}\right] \quad \text { and } \quad \partial_{v}\left(\left[u_{1}\right] \cdots\left[u_{n}\right]\right)=0 .
$$

On the other hand, there are residue homomorphisms

$$
\partial_{v}: \mathrm{W}(k(t)) \rightarrow \mathrm{W}(\kappa(v))
$$

between Witt groups determined by the formulas

$$
\partial_{v}\left\langle\pi_{v} u\right\rangle=\langle\bar{u}\rangle \quad \text { and } \quad \partial_{v}\langle u\rangle=0
$$


(see Husemoller-Milnor [18, IV, Section 1]). Recalling that $\eta[u]=\langle u\rangle-\langle 1\rangle$, we see that the following diagram commutes:

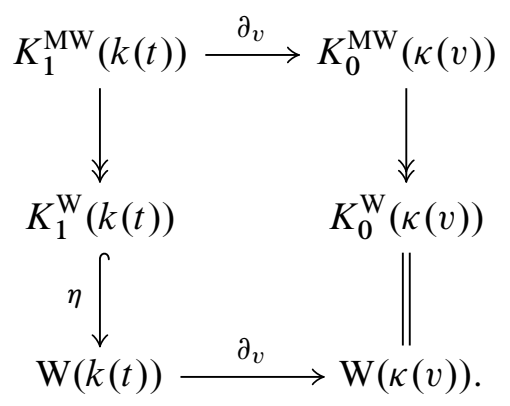

By [20, Theorem 3.24], the map

$$
\partial=\left(\partial_{v}\right)_{v \neq \infty}: K_{1}^{\mathrm{MW}}(k(t)) \rightarrow \bigoplus_{v \neq \infty} K_{0}^{\mathrm{MW}}(\kappa(v))
$$

is surjective, where the sum is taken over all finite places $v$. Given $v$ and $b \in$ $K_{0}^{\mathrm{MW}}(\kappa(v))=\mathrm{GW}(\kappa(v))$, choose $\hat{b} \in K_{1}^{\mathrm{MW}}(k(t))$ such that $\partial(\hat{b})=b$ (in particular, $\partial_{w}(\hat{b})=0$ for $\left.w \notin\{v, \infty\}\right)$. By the reciprocity formula for Morel's geometric transfers [20, (4.8)], we have

$$
\tau_{v}^{\text {geom }}(b)=-\partial_{\infty}(\hat{b})
$$

We must therefore show that

$$
\tau_{v}^{\mathrm{Sch}}(b)=-\partial_{\infty}(\hat{b}) .
$$

Since $\tau_{v}^{\text {geom }}(b)$ and $\tau_{v}^{\text {Sch }}(b)$ are both of rank $\operatorname{deg}(v) \cdot \operatorname{rk}(b),(5-12)$ shows that both sides of (5-13) have the same rank. In view of the cartesian square

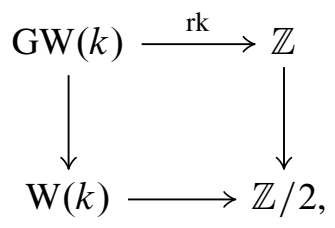

it remains to prove that (5-13) holds in the Witt group W( $k)$. By Scharlau's reciprocity theorem for Witt groups [25, Theorem 4.1] and (5-11), we have

$$
\tau_{v}^{\mathrm{Sch}}(b)=\tau_{v}^{\mathrm{Sch}} \partial_{v}(\eta \hat{b})=-\partial_{\infty}(\eta \hat{b})=-\partial_{\infty}(\widehat{b})
$$

in $\mathrm{W}(k)$, as was to be shown. There are two points to be made about the statement of the reciprocity theorem in [25]. First, the minus sign in front of $\partial_{\infty}$ does not appear 
in [25], but it appears here because we used the uniformizer $-1 / t$ instead of $1 / t$ at $\infty$, and we have $\langle-1 / t\rangle=-\langle 1 / t\rangle$ in $\mathrm{W}(k(t))$. Second, it is assumed there that char $k \neq 2$, but it was observed in [10, Section 2] that, when the Witt group is defined using symmetric bilinear forms instead of quadratic forms, the proof works in arbitrary characteristic.

\section{Appendix A: On the purity isomorphism}

In this appendix we achieve two goals:

- We show that the purity isomorphism defined by Ayoub [3, Section 1.6] is the stabilization of the one defined by Morel and Voevodsky [21, Theorem 2.23].

- We prove that the purity isomorphism $\Pi: s^{!} p^{*} \simeq \Sigma^{-\mathcal{N}_{s}} q^{*}$ is natural in the closed immersion $s$.

The naturality of $\Pi$ plays a central role in the proof of Theorem 1.3 in Section 4.

We start by recalling the definition of the Morel-Voevodsky purity zig-zag. Let $C$ be the open subscheme of the blowup of $X \times \mathbb{A}^{1}$ along $Z \times\{0\}$ whose closed complement is the blowup of $X \times\{0\}$ along $Z \times\{0\}$. We then have canonical isomorphisms

$$
C \times_{\mathbb{A}^{1}}\{0\} \simeq \mathbb{V}\left(\mathcal{N}_{S}\right) \text { and } C \times_{\mathbb{A}^{1}}\{1\} \simeq X
$$

(see Fulton [9, Chapter 5]) and diagrams
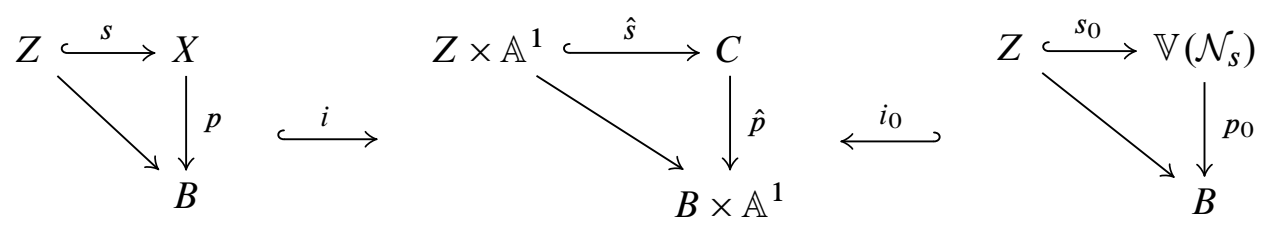

where $i$ (resp. $i_{0}$ ) is the inclusion of the fiber over 1 (resp. over 0 ). Note that $\Sigma^{-\mathcal{N}_{s}} s^{*} p^{*} \simeq s_{0}^{!} p_{0}^{*}$ by definition of $\Sigma^{-\mathcal{N}_{s}}$. Denote by $r: B \times \mathbb{A}^{1} \rightarrow B$ and $r: Z \times \mathbb{A}^{1} \rightarrow$ $Z$ the projections. Since $i$ is a section of $r$, there is a transformation $r_{*} \rightarrow i^{*}$ given by

$$
r_{*} \stackrel{\eta}{\rightarrow} r_{*} i_{*} i^{*} \simeq i^{*}
$$

Let $\Pi_{1}$ be the composition

$$
r_{*} \hat{s}^{!} \hat{p}^{*} r^{*} \stackrel{(\mathrm{A}-1)}{\longrightarrow} i^{*} \hat{s}^{!} \hat{p}^{*} r^{*} \stackrel{E x^{*} !}{\longrightarrow} s^{!} i^{*} \hat{p}^{*} r^{*} \simeq s^{!} p^{*},
$$


and let $\Pi_{0}: r_{*} \hat{s}^{!} \hat{p}^{*} r^{*} \rightarrow s_{0}^{!} p_{0}^{*}$ be the analogous composition with $i$ replaced by $i_{0}$, so that we have a zig-zag

$$
s^{!} p^{*} \stackrel{\Pi_{1}}{\longleftarrow} r_{*} \hat{s}^{!} \hat{p}^{*} r^{*} \stackrel{\Pi_{0}}{\longrightarrow} s_{0}^{!} p_{0}^{*} .
$$

Proposition A.3 The transformations $\Pi_{1}$ and $\Pi_{0}$ are isomorphisms and the composition $\Pi_{0} \Pi_{1}^{-1}$ coincides with the purity isomorphism $\Pi$.

Proof We will show that both maps in (A-2) are isomorphisms. Consider the diagram:

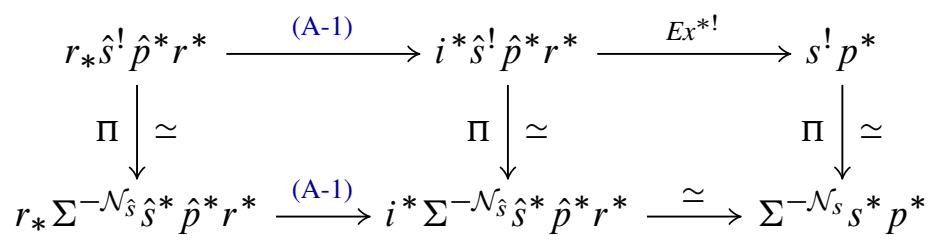

The first square commutes by naturality of the transformation (A-1) and the second square commutes by [3, Corollaire 1.6.23]. Moreover, the transformation at the bottom left is an isomorphism because $\mathcal{N}_{\hat{s}} \simeq r^{*}\left(\mathcal{N}_{s}\right)$ and (A-1) $r^{*}$ is an isomorphism. Using that $\eta$ : id $\rightarrow r_{*} r^{*}$ is an isomorphism, we see that the lower row does not change if we replace $i$ by $i_{0}$. Together with the analogous diagram for $\Pi_{0}$, we therefore obtain a commutative square:

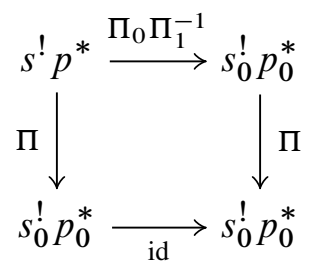

But the right-hand purity isomorphism $\Pi: s_{0}^{!} p_{0}^{*} \simeq s_{0}^{!} p_{0}^{*}$ is the identity by [3, Proposition 1.6.28], and hence $\Pi=\Pi_{0} \Pi_{1}^{-1}$, as claimed.

Proposition A.4 (Purity is natural) Suppose given a cartesian square

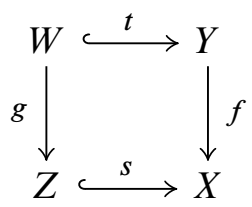

in $\mathrm{Sm}_{B}$ where $s$ and $t$ are closed immersions, and let $p: X \rightarrow B$ be the structure map. Then the induced map $\psi: g^{*}\left(\mathcal{N}_{s}\right) \rightarrow \mathcal{N}_{t}$ is an epimorphism and the diagrams 


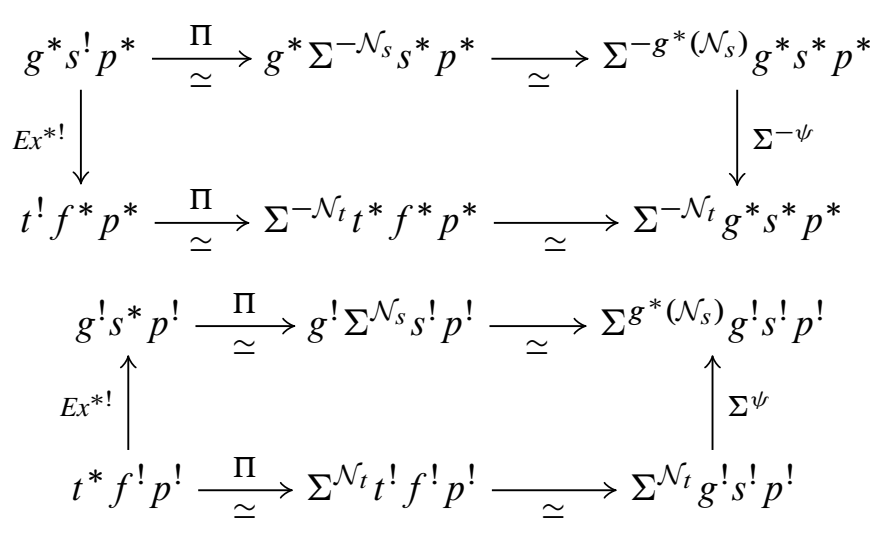

are commutative.

Remark A.5 When $f$ is smooth (in which case $\psi$ is an isomorphism), Proposition A.4 is exactly [3, Proposition 1.6.20], but in Section 4 we need the proposition for $f$ a closed immersion.

Proof Let $\mathcal{I} \subset \mathcal{O}_{X}$ be the defining ideal of $s$ and $\mathcal{J} \subset \mathcal{O}_{Y}$ that of $t$. The morphism $\psi$ is then the composition

$$
g^{*}\left(\mathcal{N}_{s}\right) \simeq g^{*} s^{*}(\mathcal{I}) \simeq t^{*} f^{*}(\mathcal{I}) \rightarrow t^{*}(\mathcal{J}) \simeq \mathcal{N}_{t}
$$

Because the square is cartesian, $\mathcal{J}$ is exactly the image of $f^{*}(\mathcal{I}) \rightarrow \mathcal{O}_{Y}$, and since $t^{*}$ is right exact, $\psi$ is an epimorphism.

We will only prove the commutativity of the first diagram; the commutativity of the second diagram is checked by a dual argument. Let $D$ be the open subscheme of the blowup of $Y \times \mathbb{A}^{1}$ along $W \times\{0\}$ whose complement is the proper transform of $Y \times\{0\}$. The given cartesian square then induces cartesian squares
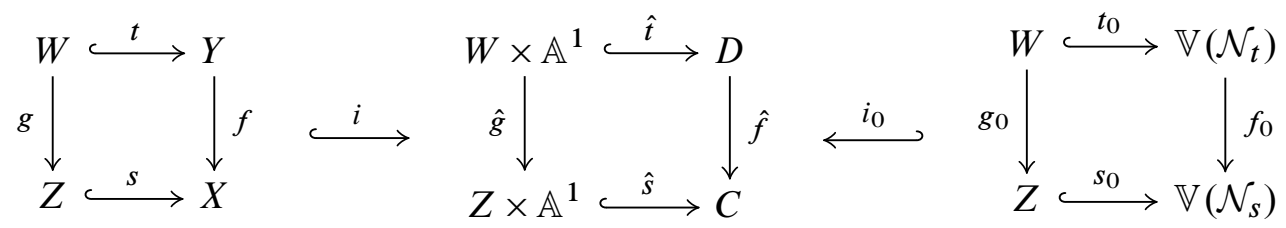

where $f_{0}=\mathbb{V}(\psi)$ and $g_{0}=g$. By Lemma B.6, the transformation $\Sigma^{-\psi} g^{*} s^{*} p^{*}$ can be identified with the exchange transformation $E x^{* !}: g_{0}^{*} s_{0}^{!} p_{0}^{*} \rightarrow t_{0}^{!} f_{0}^{*} p_{0}^{*}$. By replacing both occurrences of $\Pi$ by $\Pi_{0} \Pi_{1}^{-1}$ (Proposition A.3) and completing the resulting diagram with exchange transformations of the form $E x^{* !}$, we are reduced to proving 
the commutativity of the rectangle

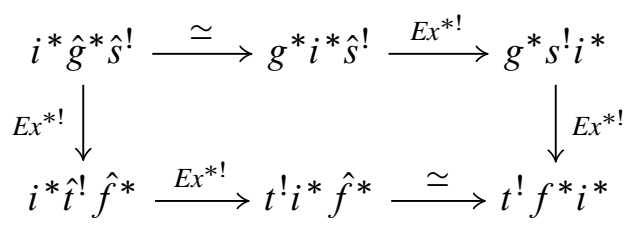

and of the analogous rectangle with $i$ replaced by $i_{0}$. By formal properties of exchange transformations [3, Définition 1.2.1], both compositions in this rectangle are equal to

$$
i^{*} \hat{g}^{*} s^{!} \stackrel{c}{\simeq}(\hat{g} i)^{*} s^{!}=(i g)^{*} s^{!} \stackrel{E x^{* !}}{\longrightarrow} t !(\text { if })^{*} \stackrel{c}{\simeq} t^{!} f^{*} i^{*} .
$$

\section{Appendix B: Coherence lemmas}

Lemma B.1 Let

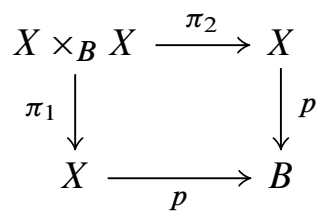

be a cartesian square of schemes and let $\delta: X \hookrightarrow X \times_{B} X$ be the diagonal. Then:

(1) The counit $\epsilon: p^{*} p_{*} \rightarrow$ id coincides with the composition

$$
p^{*} p_{*} \stackrel{E x_{*}^{*}}{\longrightarrow} \pi_{2 *} \pi_{1}^{*} \stackrel{\eta}{\rightarrow} \pi_{2 *} \delta_{*} \delta^{*} \pi_{1}^{*} \simeq \mathrm{id} .
$$

(2) If $p$ is separated of finite type, then the unit $\eta$ : id $\rightarrow p^{!} p$ ! coincides with the composition

$$
\mathrm{id} \simeq \pi_{1 !} \delta_{!} \delta ! \pi_{2}^{!} \stackrel{\epsilon}{\rightarrow} \pi_{1 !} \pi_{2}^{!} \stackrel{E x_{!}^{!}}{\longrightarrow} p ! p^{!}
$$

Proof The diagram

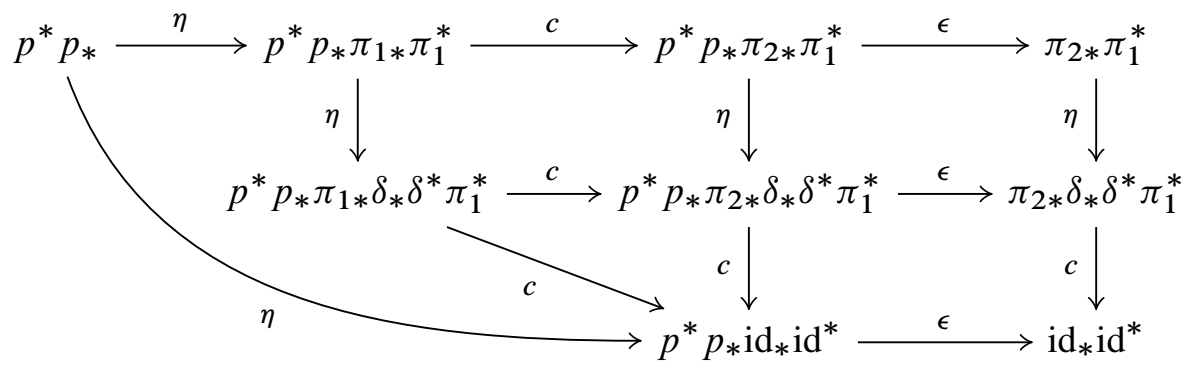

is clearly commutative. Comparing the two outer compositions proves (1). The proof of (2) is identical. 
Lemma B.2 Let

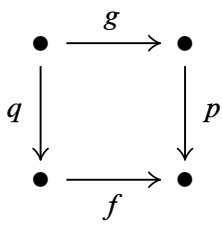

be a cartesian square of schemes where $p$ is proper and $f$ is smooth. Then the following diagram commutes:

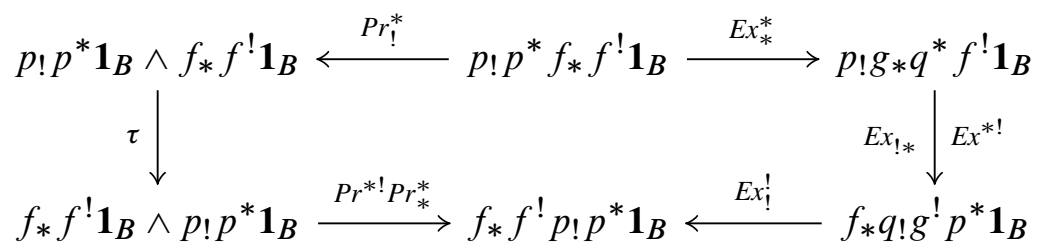

Proof Since $p$ is proper and $f$ is smooth, we can eliminate the shrieks to obtain the equivalent rectangle:

$$
\begin{array}{r}
p_{*} p^{*} \mathbf{1}_{B} \wedge f_{*} \Sigma^{\Omega_{f}} f^{*} \mathbf{1}_{B} \stackrel{P r_{*}^{*}}{\longrightarrow} p_{*} p^{*} f_{*} \Sigma^{\Omega_{f}} f^{*} \mathbf{1}_{B} \stackrel{E x_{*}^{*}}{\longrightarrow} p_{*} g_{*} \Sigma^{\Omega} q^{*} f^{*} \mathbf{1}_{B} \\
\downarrow \\
\downarrow \\
f_{*} \Sigma^{\Omega_{f}} f^{*} \mathbf{1}_{B} \wedge p_{*} p^{*} \mathbf{1}_{B} \stackrel{\operatorname{Pr}_{*}^{*}}{\longrightarrow} f_{*} \Sigma^{\Omega_{f}} f^{*} p_{*} p^{*} \mathbf{1}_{B} \stackrel{E x_{* *}}{\downarrow} f_{*} q_{*} \Sigma^{\Omega_{g}} g^{*} p^{*} \mathbf{1}_{B}
\end{array}
$$

This is now a special case of the following diagram, for $E=p^{*} \mathbf{1}_{B}$ and $F=\Sigma^{\Omega_{f}} f^{*} \mathbf{1}_{B}$ :

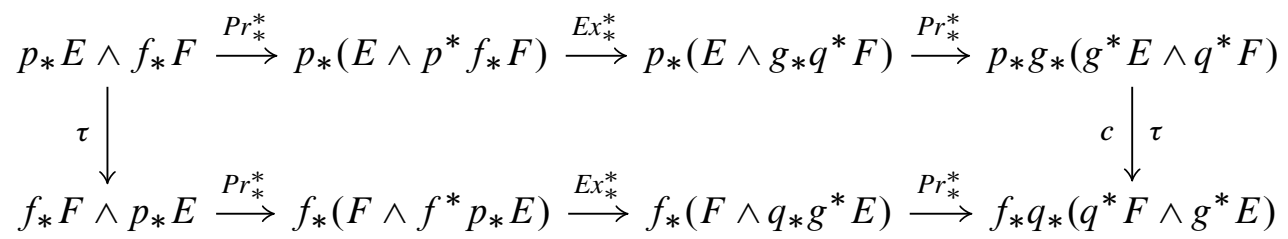

We will prove that this more general rectangle is commutative. Using the adjunction $(f q)^{*} \dashv(f q)_{*}$ and the fact that the functors $(-)^{*}$ are symmetric monoidal, it is equivalent to prove the commutativity of the following rectangle:

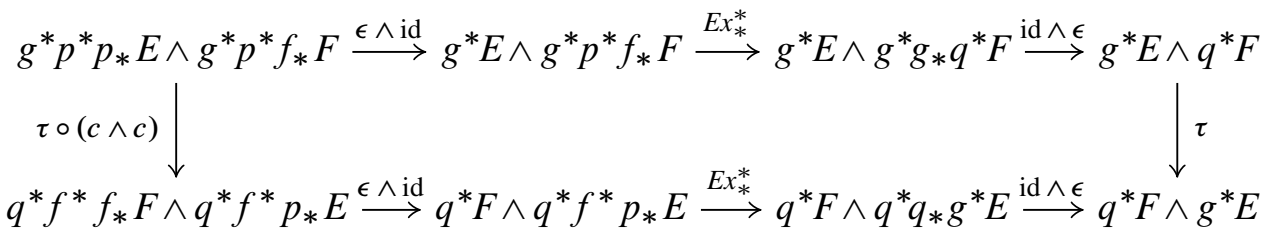


The compositions in this rectangle are now of the form $\tau \circ(\phi \wedge \psi)$ and $\left(\psi^{\prime} \wedge \phi^{\prime}\right) \circ \tau$, and hence we need only check that $\phi=\phi^{\prime}$ and $\psi=\psi^{\prime}$, ie that the squares
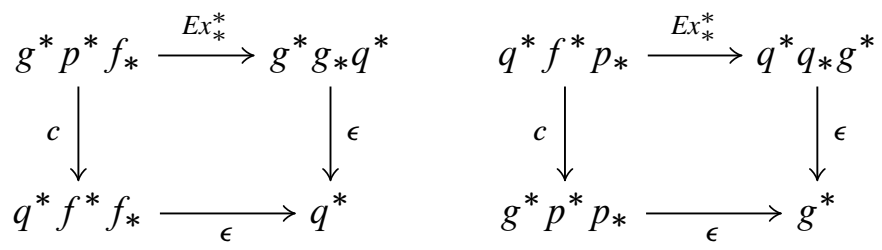

are commutative. This follows from [3, Proposition 1.2.5].

\section{Lemma B.3 Let}

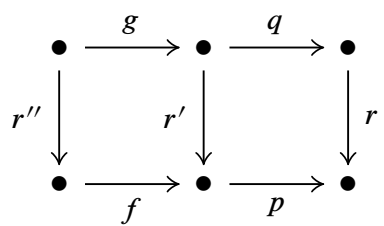

be cartesian squares in which all maps are separated of finite type. Then the following rectangle commutes:

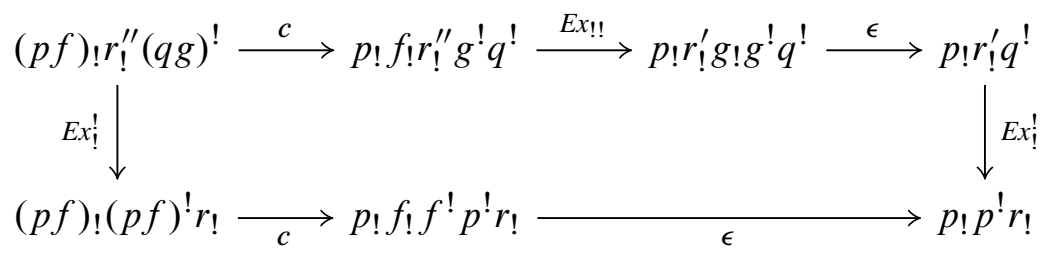

Proof We break up this rectangle as follows:

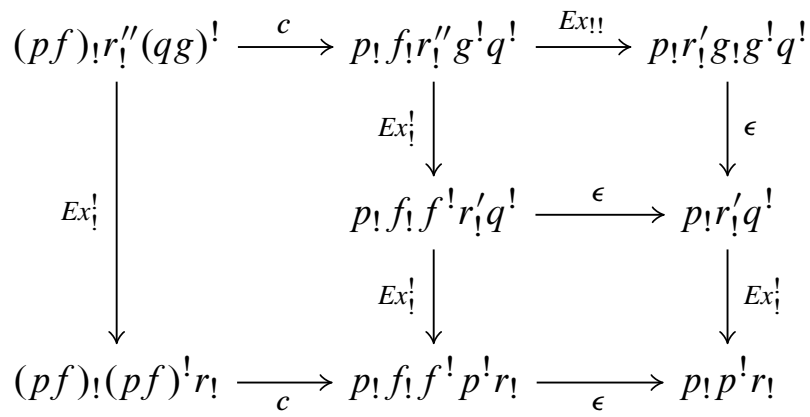

The left rectangle commutes by the compatibility of exchange transformations with the composition of cartesian squares [3, Définition 1.2.1], the top square commutes by [3, Proposition 1.2.5], and the bottom square commutes by naturality of $\epsilon$. 
Lemma B.4 Let $\gamma, \delta: X \hookrightarrow Y$ be a pair of closed immersions with a common retraction $\pi: Y \rightarrow X$ and let

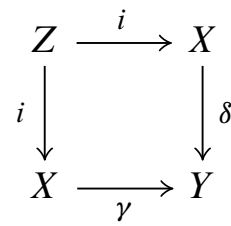

be a cartesian square. Then the composition

$$
i_{*} i \stackrel{E x_{*}^{!}}{\longrightarrow} \gamma^{!} \delta_{*} \simeq \pi_{*} \gamma_{*} \gamma^{!} \delta_{*} \stackrel{\epsilon}{\rightarrow} \pi_{*} \delta_{*} \simeq \mathrm{id}
$$

is equal to the counit $\epsilon: i_{*} i^{!} \rightarrow \mathrm{id}$.

Proof Consider the commutative diagram

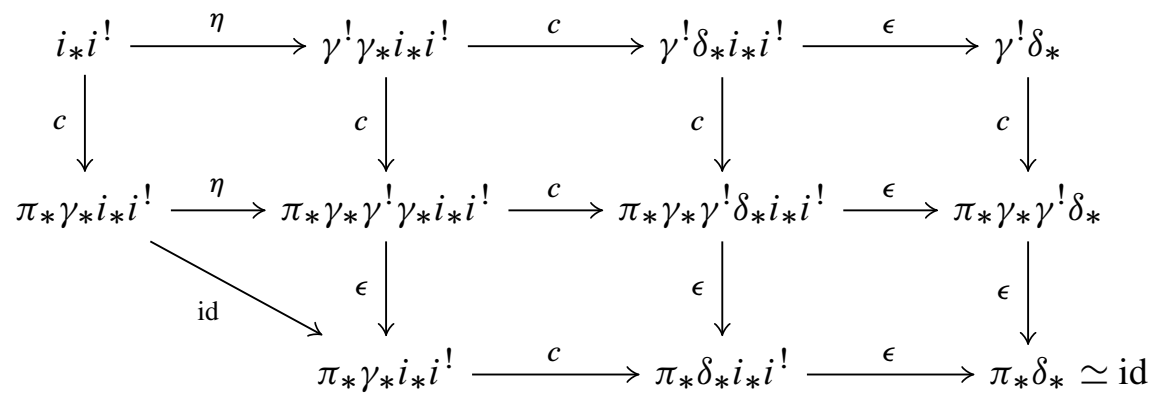

in which the upper composition is the given one. By coherence of the connection isomorphisms, the lower composition is the counit $\epsilon: i_{*} i ! \rightarrow \mathrm{id}$, which proves the lemma.

Lemma B.5 Let $i$ be a closed immersion. Then the triangle

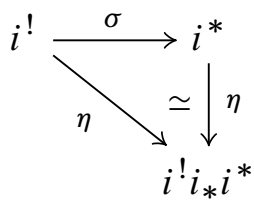

is commutative.

Proof This is simply a matter of unwinding the definitions. Recall that $\sigma$ is

$$
E x^{* !}: \mathrm{id}^{*} i^{!} \rightarrow \mathrm{id}^{\prime} i^{*} .
$$


By definition of $E x^{* !}$, this is the composition

$$
\mathrm{id}^{*} i \stackrel{\eta}{\rightarrow} \mathrm{id}^{*} i ! i_{*} i^{*} \stackrel{E x_{*}^{!}}{\longleftarrow} \mathrm{id}^{*} \mathrm{id}_{*} \mathrm{id} i^{*} \stackrel{\epsilon}{\rightarrow} \mathrm{id}^{\prime} i^{*}
$$

By construction, $E x_{*}^{!}: \mathrm{id}_{*} \mathrm{id}^{!} \rightarrow i i_{*}$ is the mate of $E x_{*}^{*}: i^{*} i_{*} \rightarrow \mathrm{id}_{*} \mathrm{id}^{*}$. Finally, by definition of $E x_{*}^{*}$, the latter is the counit $\epsilon: i^{*} i_{*} \rightarrow \mathrm{id}$, whose mate is $\eta$ : id $\rightarrow i ! i_{*}$.

\section{Lemma B.6 Let}

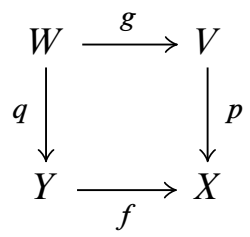

be a commutative diagram, where $p$ and $q$ are vector bundles with zero sections $s$ and $t$ and where $g$ induces a monomorphism of vector bundles $\phi: W \hookrightarrow f^{*} V$. Then the following diagrams commute (the second assuming that $f$ is separated of finite type):

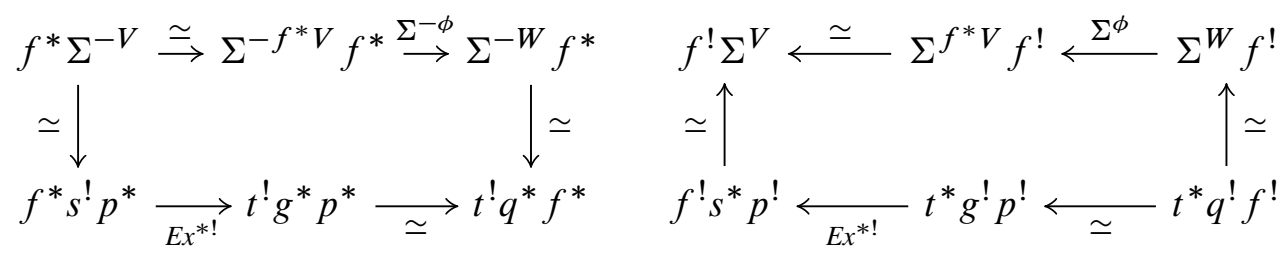

Proof Let $r: f^{*} V \rightarrow Y$ be the pullback of $p$ and let $u$ be the zero section of $r$. Recall that $\Sigma^{-\phi}$ is the composition

$$
u^{!} r^{*} \stackrel{c}{\simeq} t^{!} \phi^{!} r^{*} \stackrel{\sigma}{\rightarrow} t^{!} \phi^{*} r^{*} \stackrel{c}{\simeq} t^{!} q^{*}
$$

and that $\sigma: \phi^{!} \rightarrow \phi^{*}$ is the exchange transformation $E x^{* !}:$ id $^{*} \phi^{!} \rightarrow$ id $^{!} \phi^{*}$. The commutativity of the first rectangle then follows from the compatibility of the exchange transformation $E x^{* !}$ with the composition of the following three cartesian squares:

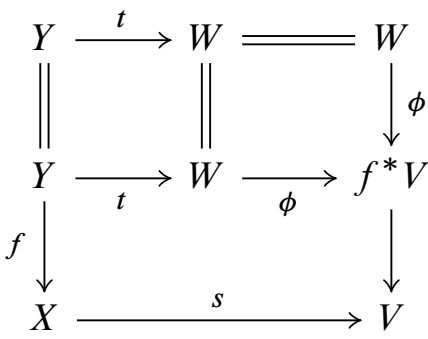

The commutativity of the second square is checked in the same way. 


\section{Appendix C: Elimination of noetherian hypotheses}

In the foundational paper [21], Morel and Voevodsky define unstable motivic homotopy theory only for noetherian schemes of finite Krull dimension. In this appendix we indicate how to properly extend the theory to arbitrary schemes. For simplicity, we will give our definitions using the language of $\infty$-categories (see Lurie [16]). We say that a scheme or a morphism of schemes is coherent if it is quasi-compact and quasi-separated.

There are two issues that arise when dropping the assumption that schemes are noetherian and finite-dimensional. The first concerns the definition of the Nisnevich topology. This topology was originally defined by Nisnevich [22] using the following pretopology: a family $\left\{U_{i} \rightarrow X\right\}_{i \in I}$ is a cover if each $U_{i} \rightarrow X$ is étale and every morphism Spec $k \rightarrow X$ with $k$ a field lifts to $U_{i}$ for some $i \in I$. For noetherian schemes, it was shown in [21, Proposition 3.1.4] that this topology is generated by a cdstructure in the sense of [29, Section 2]. For coherent schemes that are not noetherian, the pretopology and the cd-structure define different topologies, both finer than the Zariski topology and coarser than the étale topology. We will define the Nisnevich topology in general by combining the cd-structure and the Zariski topology. This choice ensures that the "small" Nisnevich $\infty$-topos $X_{\text {Nis }}$ of a scheme $X$ (ie the $\infty$-category of Nisnevich sheaves of spaces on étale $X$-schemes) has good formal properties. For instance:

(1) If $X$ is coherent, then $X_{\mathrm{Nis}}$ is coherent and compactly generated by finitely presented étale $X$-schemes.

(2) If $X$ is the limit of a cofiltered diagram of coherent schemes $X_{\alpha}$ with affine transition maps, then $X_{\mathrm{Nis}}$ is the limit of the $\infty$-topoi $\left(X_{\alpha}\right)_{\mathrm{Nis}}$.

Another point in favor of our definition is that algebraic $K$-theory, considered as a presheaf of spaces on coherent schemes, is only known to be a sheaf for our version of the Nisnevich topology. Note that property (2) determines the $\infty$-topos $X_{\mathrm{Nis}}$ for $X$ coherent once it has been defined for $X$ noetherian, since any coherent scheme is a cofiltered limit of schemes of finite type over $\mathbb{Z}$ (see Thomason-Trobaugh [27, Appendix C]). The second issue is that the Nisnevich $\infty$-topos of a coherent scheme which is not noetherian and finite-dimensional need not be hypercomplete, ie Nisnevich descent for a presheaf of spaces does not imply Nisnevich hyperdescent. We do not want to restrict ourselves to hypercomplete sheaves, since by doing so we might lose properties (1) and (2) as well as the representability of algebraic $K$-theory.

In this appendix, a presheaf is by default a presheaf of spaces. If $\mathcal{C}$ is a (possibly large) $\infty$-category, we denote by $\operatorname{PSh}(\mathcal{C})$ the $\infty$-category of presheaves on $\mathcal{C}$. It will 
be convenient to work with a weakening of the notion of topology: a quasi-topology $\tau$ on an $\infty$-category $\mathcal{C}$ assigns to every $X \in \mathcal{C}$ a collection $\tau(X)$ of sieves on $X$, called $\tau$-sieves, such that, for every $f: Y \rightarrow X, f^{*} \tau(X) \subset \tau(Y)$. A presheaf $F$ on $\mathcal{C}$ is a $\tau$-sheaf if, for every $X \in \mathcal{C}$ and every $R \in \tau(X)$, the restriction map $\operatorname{Map}(X, F) \rightarrow \operatorname{Map}(R, F)$ is an equivalence. We denote by $\operatorname{Shv}_{\tau}(\mathcal{C}) \subset \operatorname{PSh}(\mathcal{C})$ the full subcategory of $\tau$-sheaves. A family of morphisms $\left\{U_{i} \rightarrow X\right\}$ in $\mathcal{C}$ is called a $\tau$-cover if it generates a $\tau$-sieve.

If $\tau$ is a quasi-topology on $\mathcal{C}$, we denote by $\bar{\tau}$ the coarsest topology containing $\tau$. Our first goal is to show that $\operatorname{Shv}_{\tau}(\mathcal{C})=\operatorname{Shv}_{\bar{\tau}}(\mathcal{C})$. The following proposition is a generalization of [1, II, Proposition 2.2] to sheaves of spaces; the proof is exactly the same.

Proposition C.1 Let $\mathcal{C}$ be an $\infty$-category and let $\mathcal{E}$ be a collection of presheaves on $\mathcal{C}$. Let $\tau$ be the finest quasi-topology on $\mathcal{C}$ such that $\mathcal{E} \subset \operatorname{Shv}_{\tau}(\mathcal{C})$. Then $\tau$ is a topology.

Proof To begin with, note that $\tau$ exists: for $X \in \mathcal{C}, \tau(X)$ is the collection of sieves $R \hookrightarrow X$ such that, for every $f: Y \rightarrow X$ in $\mathcal{C}$ and every $F \in \mathcal{E}$, the map

$$
\operatorname{Map}(Y, F) \rightarrow \operatorname{Map}\left(f^{*} R, F\right)
$$

is an equivalence. To prove that $\tau$ is a topology, we must verify that, if $S \in \tau(X)$ and $R$ a sieve on $X$ such that $g^{*} R \in \tau\left(X^{\prime}\right)$ for every $g: X^{\prime} \rightarrow X$ in $S$, then $R \in \tau(X)$. Let $f: Y \rightarrow X$ be a morphism in $\mathcal{C}$ and let $F \in \mathcal{E}$. We must show that the left vertical arrow in the square

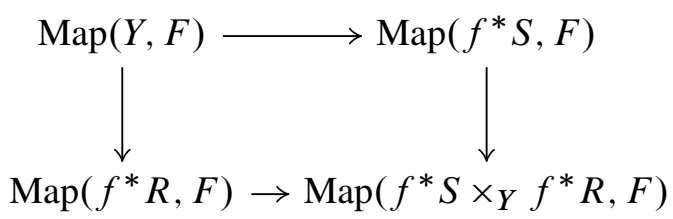

is an equivalence. We will show that the other three arrows are equivalences. The top horizontal arrow is an equivalence because $S \in \tau(X)$. For the right vertical arrow, write $f^{*} S \simeq \operatorname{colim}_{Z \in \mathcal{C} / f^{*} S} Z$ as a (possibly large) colimit of representables. Since colimits in $\operatorname{PSh}(\mathcal{C})$ are universal, $f^{*} R \times_{Y} f^{*} S \simeq \operatorname{colim}_{Z \in \mathcal{C} / f^{*} S} f^{*} R \times_{Y} Z$. For every $Z \rightarrow f^{*} S, f^{*} R \times{ }_{Y} Z$ belongs to $\tau(Z)$ by assumption, and hence

$\operatorname{Map}\left(f^{*} S, F\right) \simeq \lim _{Z} \operatorname{Map}(Z, F) \simeq \lim _{Z} \operatorname{Map}\left(f^{*} R \times_{Y} Z, F\right) \simeq \operatorname{Map}\left(f^{*} R \times_{Y} f^{*} S, F\right)$.

The proof that the bottom horizontal arrow is an equivalence is similar: write $f^{*} R \simeq$ $\operatorname{colim}_{Z \in \mathcal{C} / f^{*} R} Z$ and use that $f^{*} S \in \tau(Y)$. 
Corollary C.2 Let $\mathcal{C}$ be an $\infty$-category and $\tau$ a quasi-topology on $\mathcal{C}$. Then

$$
\operatorname{Shv}_{\tau}(\mathcal{C})=\operatorname{Shv}_{\bar{\tau}}(\mathcal{C}) .
$$

Proof Note that $\operatorname{Shv}_{\bar{\tau}}(\mathcal{C}) \subset \operatorname{Shv}_{\tau}(\mathcal{C})$. Let $\rho$ be the finest quasi-topology on $\mathcal{C}$ such that $\operatorname{Shv}_{\tau}(\mathcal{C}) \subset \operatorname{Shv}_{\rho}(\mathcal{C})$. Tautologically, $\rho$ contains $\tau$. By Proposition C.1, $\rho$ contains $\bar{\tau}$. Hence, $\operatorname{Shv}_{\rho}(\mathcal{C}) \subset \operatorname{Shv}_{\bar{\tau}}(\mathcal{C})$.

We also need an easy-to-use version of the "comparison lemma" [1, III, Théorème 4.1] for sheaves of spaces:

Lemma C.3 Let $\mathcal{D}$ be an $\infty$-category, $\mathcal{C}$ a small $\infty$-category and $u: \mathcal{C} \hookrightarrow \mathcal{D}$ a fully faithful functor. Let $\tau$ and $\rho$ be quasi-topologies on $\mathcal{C}$ and $\mathcal{D}$, respectively. Suppose that:

(a) Every $\tau$-sieve is generated by a cover $\left\{U_{i} \rightarrow X\right\}$ such that:

(a1) The fiber products $U_{i_{0}} \times_{X} \cdots \times_{X} U_{i_{n}}$ exist and are preserved by $u$.

(a2) $\left\{u\left(U_{i}\right) \rightarrow u(X)\right\}$ is a $\bar{\rho}$-cover $\mathcal{D}$.

(b) For every $X \in \mathcal{C}$ and every $\rho$-sieve $R \hookrightarrow u(X), u^{*}(R) \hookrightarrow X$ is a $\bar{\tau}$-sieve in $\mathcal{C}$.

(c) Every $X \in \mathcal{D}$ admits a $\bar{\rho}$-cover $\left\{U_{i} \rightarrow X\right\}$ such that the fiber products $U_{i_{0}} \times_{X}$ $\cdots \times{ }_{X} U_{i_{n}}$ exist and belong to the essential image of $u$.

Then the adjunction $u^{*} \dashv u_{*}$ restricts to an equivalence of $\infty$-categories $\operatorname{Shv}_{\rho}(\mathcal{D}) \simeq$ $\operatorname{Shv}_{\tau}(\mathcal{C})$.

We can rephrase the conclusion of the lemma as follows: a presheaf on $\mathcal{D}$ is a $\rho$-sheaf if and only if it is the right Kan extension of a $\tau$-sheaf on $\mathcal{C}$. An immediate consequence of the lemma is that the inclusion $\operatorname{Shv}_{\rho}(\mathcal{D}) \subset \operatorname{PSh}(\mathcal{D})$ admits a left exact left adjoint $a_{\rho}$, namely the composition $u_{*} a_{\tau} u^{*}$.

Proof We tacitly use Corollary C. 2 throughout the proof. We first show that $u^{*}$ and $u_{*}$ preserve sheaves. Let $\mathfrak{U}$ be a $\tau$-cover as in (a) and let

$$
\check{C}(\mathfrak{U}) \in \operatorname{Fun}\left(\Delta^{\mathrm{op}}, \operatorname{PSh}(\mathcal{C})\right)
$$

be its $\check{C}$ ech nerve (note that colim $\check{C}(\mathfrak{U})$ is the sieve generated by $\mathfrak{U})$. By (a1), $u$ ! $\check{C}(\mathfrak{U}) \simeq$ $\check{C}(u(\mathfrak{U}))$, and by (a2), $u(\mathfrak{U})$ is a $\bar{\rho}$-cover. If $F$ is a $\rho$-sheaf, we deduce that

$$
\operatorname{Map}\left(u_{!} X, F\right) \rightarrow \operatorname{Map}(u ! \operatorname{colim} \check{C}(\mathfrak{U}), F)
$$

is an equivalence. By adjunction, $u^{*}$ preserves sheaves. Let $X \in \mathcal{D}$ and let $R \hookrightarrow X$ be a $\rho$-sieve. We claim that $u^{*}(R) \hookrightarrow u^{*}(X)$ becomes an equivalence after $\tau$-sheafification. 
By the universality of colimits in $\operatorname{PSh}(\mathcal{C})$, it suffices to show that, for every $Y \in \mathcal{C}$ and every morphism $u(Y) \rightarrow X, u^{*}\left(R \times_{X} u(Y)\right) \hookrightarrow Y$ is a $\bar{\tau}$-sieve. This follows from (b) since $R \times_{X} u(Y)$ is a $\rho$-sieve. By adjunction, $u_{*}$ preserves sheaves. Thus, the adjunction $u^{*} \dashv u_{*}$ restricts to an adjunction

$$
u^{*}: \operatorname{Shv}_{\rho}(\mathcal{D}) \rightleftarrows \operatorname{Shv}_{\tau}(\mathcal{C}): u_{*},
$$

where $u_{*}$ is fully faithful. It remains to show that $u^{*}$ is conservative on $\operatorname{Shv}_{\rho}(\mathcal{D})$, but this follows at once from (c).

A cartesian square of schemes

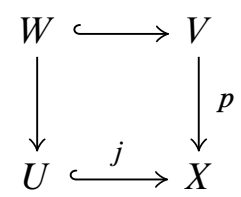

will be called a Nisnevich square over $X$ if $j$ is an open immersion, $p$ is étale and there exists a closed immersion $Z \hookrightarrow X$ complement to $U$ such that $p$ induces an isomorphism $V \times_{X} Z \simeq Z$. We say that such a square is finitely presented if $j$ and $p$ are finitely presented.

Let $B$ be a scheme. We denote by $\mathrm{Sm}_{B}$ the category of smooth $B$-schemes and by $\mathrm{Sm}_{B}^{\prime} \subset \mathrm{Sm}_{B}$ the full subcategory spanned by compositions of open immersions and finitely presented smooth morphisms. If $B$ is coherent, we also consider the subcategory $\mathrm{Sm}_{B}^{\mathrm{fp}} \subset \mathrm{Sm}_{B}$ of finitely presented smooth $B$-schemes. We will define the following quasi-topologies on $\mathrm{Sm}_{B}$ :

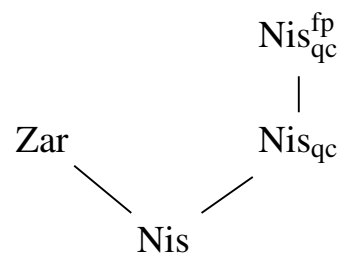

The quasi-topology Zar will also be defined on $\mathrm{Sm}_{B}^{\prime}$, and $\mathrm{Nis}_{\mathrm{qc}}^{\mathrm{fp}}$ and Nis will also be defined on $\mathrm{Sm}_{B}^{\prime}$ and $\mathrm{Sm}_{B}^{\mathrm{fp}}$. The Zar-sieves are the sieves generated by open covers. The quasi-topology $\mathrm{Nis}_{\mathrm{qc}}$ (resp. $\mathrm{Nis}_{\mathrm{qc}}^{\mathrm{fp}}$ ) consists of:

- The empty sieve on $\varnothing$.

- For every Nisnevich square (resp. finitely presented Nisnevich square) as above, the sieve generated by $\{j, p\}$. 
The Nisnevich quasi-topology Nis is then defined as follows on each category: Nis $=$ Zar $\cup \mathrm{Nis}_{\mathrm{qc}}$ on $\mathrm{Sm}_{B}$, Nis $=$ Zar $\cup \mathrm{Nis}_{\mathrm{qc}}^{\mathrm{fp}}$ on $\mathrm{Sm}_{B}^{\prime}$, and $\mathrm{Nis}=\mathrm{Nis}_{\mathrm{qc}}^{\mathrm{fp}}$ on $\mathrm{Sm}_{B}^{\mathrm{fp}}$.

Lemma C.4 For every $\mathrm{Nis}_{\mathrm{qc}}-$ sieve $R \hookrightarrow X$ in $\mathrm{Sm}_{B}$, there exists an open cover $\left\{f_{i}: X_{i} \hookrightarrow X\right\}$ such that $f_{i}^{*} R$ contains a Nis $\mathrm{qc}_{\mathrm{qc}}^{\mathrm{fp}}-$ sieve.

Proof Let $j: U \hookrightarrow X \leftarrow V: p$ be a Nisnevich square generating $R$ and let $Z$ be a closed complement of $j$ such that $V \times_{X} Z \simeq Z$. Taking an open cover of $X$ if necessary, we may assume that $X$ is coherent. Let $\left\{V_{i}\right\}$ be an open cover of $V$ by coherent schemes and let $X_{i}=p\left(V_{i}\right)$. Then $V_{i} \rightarrow X_{i}$ is finitely presented and is an isomorphism over $Z \cap X_{i}$. Since $\left\{U, X_{i}\right\}$ is an open cover of $X$, we may assume that $p$ is finitely presented. As $X$ is coherent, we can write $Z=\lim _{\alpha} Z_{\alpha}$ where each $Z_{\alpha}$ is a finitely presented closed subscheme of $X$. Since $p$ is finitely presented and is an isomorphism over $Z$, there exists $\alpha$ such that $p$ is an isomorphism over $Z_{\alpha}$. If $j_{\alpha}$ is the open immersion complement to $Z_{\alpha}$, then $\left\{j_{\alpha}, p\right\}$ is a $\operatorname{Nis}_{\mathrm{qc}}^{\mathrm{fp}}-$ cover refining $\{j, p\}$.

We say that a presheaf $F$ on $\mathrm{Sm}_{B}^{(\mathrm{fp})}$ satisfies Nisnevich excision if:

- $F(\varnothing) \simeq *$

- For every Nisnevich square $Q$ in $\operatorname{Sm}_{B}^{(\mathrm{fp})}, F(Q)$ is cartesian.

Proposition C.5 Let $B$ be a scheme.

(1) A presheaf on $\mathrm{Sm}_{B}$ is a Nisnevich sheaf if and only if it is the right Kan extension of a Nisnevich sheaf on $\mathrm{Sm}_{B}^{\prime}$. In particular, $\operatorname{Shv}_{\mathrm{Nis}}\left(\mathrm{Sm}_{B}\right)$ is an $\infty$-topos and the inclusion $\operatorname{Shv}_{\mathrm{Nis}}\left(\mathrm{Sm}_{B}\right) \subset \mathrm{PSh}\left(\mathrm{Sm}_{B}\right)$ admits a left exact left adjoint.

(2) A presheaf on $\mathrm{Sm}_{B}$ is a Nisnevich sheaf if and only if it satisfies Zariski descent and Nisnevich excision.

If $B$ is coherent, then:

(3) A presheaf on $\mathrm{Sm}_{B}$ is a Nisnevich sheaf if and only if it is the right Kan extension of a Nisnevich sheaf on $\operatorname{Sm}_{B}^{\mathrm{fp}}$. In particular, $\operatorname{Shv}_{\mathrm{Nis}}\left(\operatorname{Sm}_{B}\right) \simeq \operatorname{Shv}_{\mathrm{Nis}}\left(\operatorname{Sm}_{B}^{\mathrm{fp}}\right)$.

(4) A presheaf on $\mathrm{Sm}_{B}^{\mathrm{fp}}$ is a Nisnevich sheaf if and only if it satisfies Nisnevich excision. 
Proof (1) It suffices to verify the assumptions of Lemma C.3 for the inclusion $\mathrm{Sm}_{B}^{\prime} \subset \mathrm{Sm}_{B}$. The only nontrivial point is (b), which follows from Lemma C.4.

(3) By (1), it suffices to verify the assumptions of Lemma C.3 for the inclusion $\mathrm{Sm}_{B}^{\mathrm{fp}} \subset \mathrm{Sm}_{B}^{\prime}$. For (c), note that every scheme in $\mathrm{Sm}_{B}^{\prime}$ is quasi-separated.

$(2,4)$ For $Q$ a Nisnevich square

$$
j: U \hookrightarrow X \leftarrow V: p
$$

in $\operatorname{Sm}_{B}^{(\mathrm{fp})}$, denote by $C_{Q} \in \operatorname{PSh}\left(\mathrm{Sm}_{B}^{(\mathrm{fp})}\right)$ the colimit of the $\check{C}$ ech nerve $\check{C}(\{j, p\})$ (ie the sieve generated by $\{j, p\})$ and by

$$
K_{Q} \in \operatorname{PSh}\left(\operatorname{Sm}_{B}^{(\mathrm{fp})}\right)
$$

the pushout of $Q$. Let $C$ (resp. $K$ ) be the class of morphisms of the form $C_{Q} \rightarrow$ $X$ (resp. $\left.K_{Q} \rightarrow X\right)$ in $\operatorname{PSh}\left(\operatorname{Sm}_{B}^{(\mathrm{fp})}\right)$, where $Q$ is any Nisnevich square, together with the empty sieve on the empty scheme. By definition, a presheaf is a $\mathrm{Nis}_{\mathrm{qc}}^{(\mathrm{fp})}-$ sheaf if and only if it is $C$-local and it satisfies Nisnevich excision if and only if it is $K$-local. The arguments of [29, Section 5] show that $C$ and $K$ generate the same class of morphisms under 2-out-of-3, pushouts and colimits, whence the result.

These technical preliminaries aside, we can now define the unstable motivic homotopy category $\mathrm{H}(B)$ of an arbitrary scheme $B$. We say that a presheaf $F$ on $\operatorname{Sm}_{B}$ is $\mathbb{A}^{1}$ invariant if, for every $X \in \mathrm{Sm}_{B}$, the projection $\mathbb{A}^{1} \times X \rightarrow X$ induces an equivalence $F(X) \simeq F\left(\mathbb{A}^{1} \times X\right)$. Note that if $F$ is Nisnevich sheaf on $\mathrm{Sm}_{B}$, the $\mathbb{A}^{1}$-invariance condition can be checked on $\mathrm{Sm}_{B}^{\prime}$, and even on $\mathrm{Sm}_{B}^{\text {fp }}$ if $B$ is coherent.

We let $\mathrm{H}(B) \subset \operatorname{Shv}_{\text {Nis }}\left(\operatorname{Sm}_{B}\right)$ be the full subcategory of $\mathbb{A}^{1}$-invariant Nisnevich sheaves. This definition is of course equivalent to the standard one when $B$ is noetherian and of finite Krull dimension. By Proposition C.5(1), $\mathrm{H}(B)$ is a presentable $\infty$-category and the inclusion $\mathrm{H}(B) \subset \mathrm{PSh}\left(\mathrm{Sm}_{B}\right)$ admits a left adjoint

$$
M: \operatorname{PSh}\left(\operatorname{Sm}_{B}\right) \rightarrow \mathrm{H}(B) .
$$

Proposition C.6 The functor $M$ preserves finite products.

Proof As $M$ factors through $\operatorname{PSh}\left(\mathrm{Sm}_{B}^{\prime}\right)$, it suffices to show that $M^{\prime}: \operatorname{PSh}\left(\mathrm{Sm}_{B}^{\prime}\right) \rightarrow$ $\mathrm{H}(B)$ preserves finite products. The functor

$$
L_{\mathbb{A}^{1}}: F \mapsto \operatorname{colim}_{n \in \Delta^{\mathrm{op}}} F\left(\mathbb{A}^{n} \times-\right)
$$

is left adjoint to the inclusion of $\mathbb{A}^{1}$-invariant presheaves into all presheaves, and it preserves finite products since $\Delta^{\mathrm{op}}$ is sifted. Let $a_{\mathrm{Nis}}$ be the Nisnevich sheafification 
functor. A standard argument shows that there exists an ordinal $\alpha$ such that the $\alpha$ th iteration of $L_{\mathbb{A}^{1}} \circ a_{\mathrm{Nis}}$, viewed as a pointed endofunctor of $\operatorname{PSh}\left(\operatorname{Sm}_{B}^{\prime}\right)$, is equivalent to $M^{\prime}$. Since $L_{\mathbb{A}^{1}}, a_{\mathrm{Nis}}$ and transfinite composition preserve finite products, so does $M^{\prime}$.

As is usual, if $X \in \mathrm{Sm}_{B}$, we will commit an abuse of notation and denote by $X$ the image of $X$ by the functor $\mathrm{Sm}_{B} \rightarrow \mathrm{H}(B)$, composition of the Yoneda embedding and the localization functor $M$.

If $f: B^{\prime} \rightarrow B$ is a morphism of schemes, the base change functor $\mathrm{Sm}_{B} \rightarrow \mathrm{Sm}_{B^{\prime}}$ preserves trivial line bundles and Čech nerves of Nisnevich covers. It follows that the functor

$$
\operatorname{PSh}\left(\operatorname{Sm}_{B^{\prime}}\right) \rightarrow \operatorname{PSh}\left(\operatorname{Sm}_{B}\right), \quad F \mapsto F\left(-\times_{B} B^{\prime}\right)
$$

preserves $\mathbb{A}^{1}$-invariant Nisnevich sheaves and hence restricts to a limit-preserving functor $f_{*}: \mathrm{H}\left(B^{\prime}\right) \rightarrow \mathrm{H}(B)$. We denote by $f^{*}$ its left adjoint; it preserves finite products by Proposition C.6. If $f$ is smooth, the base change functor $\mathrm{Sm}_{B} \rightarrow \mathrm{Sm}_{B^{\prime}}$ has a left adjoint, namely the forgetful functor $\mathrm{Sm}_{B^{\prime}} \rightarrow \mathrm{Sm}_{B}$, which also preserves trivial line bundles and Čech nerves of Nisnevich covers. It follows that in this case $f^{*}$ has a left adjoint $f_{\sharp}: \mathrm{H}\left(B^{\prime}\right) \rightarrow \mathrm{H}(B)$. We immediately verify that the exchange transformation $E x_{\sharp}^{*}$ and the projector $\operatorname{Pr}_{\sharp}^{*}$ are equivalences.

\section{Proposition C.7}

(1) If $B$ is a coherent scheme, every $X \in \operatorname{Sm}_{B}^{\text {fp }}$ is compact in $\mathrm{H}(B)$.

(2) If $f: B^{\prime} \rightarrow B$ is coherent, $f_{*}: \mathrm{H}\left(B^{\prime}\right) \rightarrow \mathrm{H}(B)$ preserves filtered colimits.

(3) If $B$ is the limit of a cofiltered diagram of coherent schemes $B_{\alpha}$ with affine transition maps, then $\mathrm{H}(B) \simeq \lim _{\alpha} \mathrm{H}\left(B_{\alpha}\right)$ in the $\infty$-category of $\infty$-categories.

Proof (1) It suffices to show that $\mathrm{H}(B)$ is closed under filtered colimits in $\operatorname{PSh}\left(\operatorname{Sm}_{B}^{\mathrm{fp}}\right)$. In fact, it is obvious that the subcategories of $\mathbb{A}^{1}$-invariant presheaves and of presheaves satisfying Nisnevich excision are both closed under filtered colimits.

(2) By Zariski descent, we can assume that $B$ is coherent. The $\infty$-category $\mathrm{H}(B)$ is then generated under colimits by $X \in \mathrm{Sm}_{B}^{\mathrm{fp}}$. By (1), these generators are compact and are carried by $f^{*}$ to compact objects in $\mathrm{H}\left(B^{\prime}\right)$. The result follows by adjunction.

(3) In this situation, the category $\operatorname{Sm}_{B}^{\mathrm{fp}}$ is the colimit of the categories $\operatorname{Sm}_{B_{\alpha}}^{\mathrm{fp}}$ and hence $\operatorname{PSh}\left(\operatorname{Sm}_{B}^{\mathrm{fp}}\right)$ is the limit of the $\infty$-categories $\operatorname{PSh}\left(\operatorname{Sm}_{B_{\alpha}}^{\mathrm{fp}}\right)$. It remains to show that

$$
F \in \operatorname{PSh}\left(\operatorname{Sm}_{B}^{\mathrm{fp}}\right)
$$


is an $\mathbb{A}^{1}$-invariant Nisnevich sheaf if, for all $\alpha$, its image in $\operatorname{PSh}\left(\operatorname{Sm}_{B_{\alpha}}^{\mathrm{fp}}\right)$ is. This follows from the fact that any trivial line bundle (resp. any Nisnevich square) in $\mathrm{Sm}_{B}^{\mathrm{fp}}$ is the pullback of a trivial line bundle (resp. a Nisnevich square) in $\operatorname{Sm}_{B_{\alpha}}^{\text {fp }}$ for some $\alpha$.

Our next goal is to generalize the gluing theorem of Morel-Voevodsky [21, Theorem 3.2.21] to our setting. The proof in [21] uses the fact that henselian local schemes form a conservative family of points of the $\infty$-topos $\operatorname{Shv}_{\mathrm{Nis}}\left(\mathrm{Sm}_{B}\right)$, which is not true anymore when $B$ is not noetherian of finite Krull dimension. While it is not difficult to give a proof of the gluing theorem that avoids the use of points and works in general, we will give instead a shorter argument that reduces the general case to the Morel-Voevodsky case.

Suppose that $B$ is a cofiltered limit of coherent schemes $B_{\alpha}$, so that $\mathrm{H}(B) \simeq \lim _{\alpha} \mathrm{H}\left(B_{\alpha}\right)$. Let $f_{\beta \alpha}: B_{\beta} \rightarrow B_{\alpha}$ be the transition maps and $f_{\alpha}: B \rightarrow B_{\alpha}$ the canonical projections. Then, by [16, Lemma 6.3.3.6],

$$
\operatorname{id}_{\mathrm{H}(B)} \simeq \operatorname{colim}_{\alpha} f_{\alpha}^{*} f_{\alpha *} .
$$

Moreover, since functors of the form $f_{*}$ preserve filtered colimits, the left adjoint functors $f_{\alpha}^{*}$ can be computed as

$$
f_{\beta *} f_{\alpha}^{*} \simeq \operatorname{colim}_{\gamma} f_{\gamma \beta *} f_{\gamma \alpha}^{*}
$$

Proposition C.10 Let $B$ be a scheme and let $i: Z \hookrightarrow B$ be a closed immersion with open complement $j: U \hookrightarrow B$. Then:

(1) For every $F \in \mathrm{H}(B)$, the square

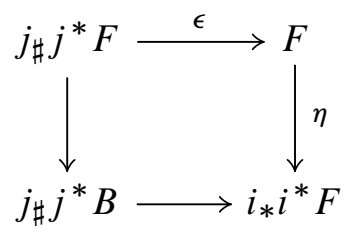

is cocartesian.

(2) $i_{*}: \mathrm{H}(Z) \rightarrow \mathrm{H}(B)$ is fully faithful.

Proof (1) By Zariski descent, we can assume that $B$ is coherent. Let $\left\{i_{\alpha}: Z_{\alpha} \hookrightarrow B\right\}$ be the cofiltered poset of finitely presented closed subschemes of $B$ containing $Z$ and let $j_{\alpha}: U_{\alpha} \hookrightarrow B$ be the open immersion complement to $i_{\alpha}$. Then $Z \simeq \lim _{\alpha} Z_{\alpha}$ and 
$\left\{U_{\alpha}\right\}$ is an open cover of $U$ which is closed under binary intersections. By Zariski descent, the canonical transformation

$$
\underset{\alpha}{\operatorname{colim}} j_{\alpha \sharp} j_{\alpha}^{*} \rightarrow j_{\sharp} j^{*}
$$

is an equivalence. On the other hand, by (C-9) and a cofinality argument, the canonical transformation

$$
\underset{\alpha}{\operatorname{colim}} i_{\alpha *} i_{\alpha}^{*} \rightarrow i_{*} i^{*}
$$

is an equivalence. It therefore suffices to prove the result when $B$ is coherent and $i$ is finitely presented. In that case, we can write $i$ and $j$ as cofiltered limits of complementary immersions $i_{\alpha}: Z_{\alpha} \hookrightarrow B_{\alpha}$ and $j_{\alpha}: U_{\alpha} \hookrightarrow B_{\alpha}$, such that $B_{\alpha}$ is of finite type over $\mathbb{Z}$ and such that the squares

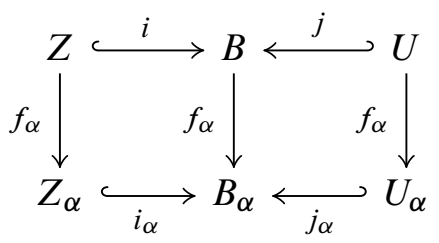

are cartesian. Let $F_{\alpha}=f_{\alpha *} F$ be the component of $F$ in $\mathrm{H}\left(B_{\alpha}\right)$, so that, by (C-8), $F \simeq \operatorname{colim}_{\alpha} f_{\alpha}^{*} F_{\alpha}$. Since $B_{\alpha}$ is noetherian of finite Krull dimension, we have a cocartesian square

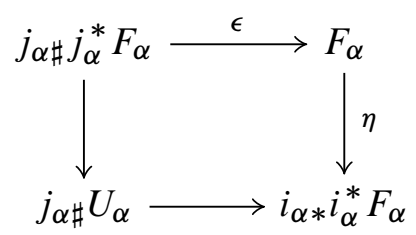

in $\mathrm{H}\left(B_{\alpha}\right)$. Applying $f_{\alpha}^{*}$ and taking the colimit over $\alpha$, we obtain a cocartesian square in $\mathrm{H}(B)$ which maps canonically to the given square. Moreover, the maps on the top left, bottom left and top right corners are equivalences since $f_{\alpha}^{*} j_{\alpha \sharp} \simeq j_{\sharp} f_{\alpha}^{*}$ and since $j_{\#}$ and $j^{*}$ preserve colimits. It remains to prove that the map

$$
\operatorname{colim}_{\alpha} f_{\alpha}^{*} i_{\alpha *} i_{\alpha}^{*} F_{\alpha} \rightarrow i_{*} i^{*} F
$$

on the bottom right corner is an equivalence. Since $i_{*}$ preserves filtered colimits, it suffices to show that the exchange transformation $E x_{*}^{*}: f_{\alpha}^{*} i_{\alpha *} \rightarrow i_{*} f_{\alpha}^{*}$ is an equivalence. Using (C-9) and the fact that the exchange transformation $f_{\gamma \alpha}^{*} i_{\alpha *} \rightarrow i_{\gamma *} f_{\gamma \alpha}^{*}$ is an equivalence (which is a consequence of the gluing theorem for finite-dimensional 
noetherian schemes), we compute

$$
\begin{aligned}
f_{\beta *} f_{\alpha}^{*} i_{\alpha *} & \simeq \operatorname{colim}_{\gamma} f_{\gamma \beta *} f_{\gamma \alpha}^{*} i_{\alpha *} \simeq \operatorname{colim}_{\gamma} f_{\gamma \beta *} i_{\gamma *} f_{\gamma \alpha}^{*} \\
& \simeq \operatorname{colim}_{\gamma} i_{\beta *} f_{\gamma \beta *} f_{\gamma \alpha}^{*} \simeq i_{\beta *} \operatorname{colim}_{\gamma} f_{\gamma \beta *} f_{\gamma \alpha}^{*} \simeq i_{\beta *} f_{\beta *} f_{\alpha}^{*} \simeq f_{\beta *} i_{*} f_{\alpha}^{*} .
\end{aligned}
$$

One verifies easily that this composition coincides with $f_{\beta *} E x_{*}^{*}$, which completes the proof.

(2) Applying (1) to $i_{*} F$, we deduce that the unit id $\rightarrow i_{*} i^{*}$ is an equivalence on $i_{*} F$. It follows from a triangle identity that the counit $i^{*} i_{*} \rightarrow$ id becomes an equivalence after applying $i_{*}$. By [12, Proposition 18.1.1], $\mathrm{H}(Z)$ is generated under colimits by pullbacks of smooth $B$-schemes. It follows that $i_{*}$ is conservative and hence fully faithful.

Denote by $\mathrm{H}_{*}(B)$ the undercategory $\mathrm{H}(B)_{B}$. All the features of $\mathrm{H}(B)$ discussed so far have obvious analogs for $\mathrm{H}_{*}(B)$. The smash product $\wedge$ on $\mathrm{H}_{*}(B)$ is the unique symmetric monoidal product which is compatible with colimits and for which the functor $(-)_{+}: \mathrm{H}(B) \rightarrow \mathrm{H}_{*}(B)$ is symmetric monoidal. One can then define the $\infty$-category $\mathrm{SH}(B)$ as a symmetric monoidal presentable $\infty$-category as in [24, Definition 4.8], by formally inverting $S^{\mathbb{A}^{1}}$ for the smash product on $\mathrm{H}_{*}(B)$. We thus have a symmetric monoidal colimit-preserving functor

$$
\Sigma^{\infty}: \mathrm{H}_{*}(B) \rightarrow \mathrm{SH}(B),
$$

and we let $\Sigma_{+}^{\infty}=\Sigma^{\infty} \circ(-)_{+}$. Note that $\operatorname{SH}(B)$ is stable since $S^{\mathbb{A}^{1}}$ is the suspension of the pointed motivic space $\left(\mathbb{A}^{1}-0,1\right)$. Because the cyclic permutation of $S^{\mathbb{A}^{1}} \wedge$ $S^{\mathbb{A}^{1}} \wedge S^{\mathbb{A}^{1}}$ is homotopic to the identity, $\mathrm{SH}(B)$ can also be described as the following limit of $\infty$-categories:

$$
\mathrm{SH}(B)=\lim \left(\cdots \stackrel{\Omega^{\mathbb{A}^{1}}}{\longrightarrow} \mathrm{H}_{*}(B) \stackrel{\Omega^{\mathbb{A}^{1}}}{\longrightarrow} \mathrm{H}_{*}(B)\right),
$$

where $\Omega^{\mathbb{A}^{1}}$ is right adjoint to $\Sigma^{\mathbb{A}^{1}}[24$, Corollary 4.24].

If $f: B^{\prime} \rightarrow B$ is a morphism of schemes, then $f_{*} \Omega^{\mathbb{A}^{1}} \simeq \Omega^{\mathbb{A}^{1}} f_{*}$ and hence $f_{*}$ induces a limit-preserving functor $f_{*}: \mathrm{SH}\left(B^{\prime}\right) \rightarrow \mathrm{SH}(B)$. Its left adjoint $f^{*}$ is the unique colimit-preserving symmetric monoidal functor $f^{*}: \mathrm{SH}(B) \rightarrow \mathrm{SH}\left(B^{\prime}\right)$ such that $f^{*} \Sigma_{+}^{\infty} X=\Sigma_{+}^{\infty}\left(X \times_{B} B^{\prime}\right)$ for $X \in \mathrm{Sm}_{B}$.

\section{Proposition C.12}

(1) $\mathrm{SH}(B)$ is generated under colimits by objects of the form $\Sigma^{-\mathbb{A}^{n}} \Sigma_{+}^{\infty} X$ for $X \in \operatorname{Sm}_{B}$ and $n \geq 0$. 
(2) If $B$ is a coherent scheme and $X \in \mathrm{Sm}_{B}^{\mathrm{fp}}, \Sigma_{+}^{\infty} X \in \mathrm{SH}(B)$ is compact.

(3) If $f: B^{\prime} \rightarrow B$ is coherent, $f_{*}: \mathrm{SH}\left(B^{\prime}\right) \rightarrow \mathrm{SH}(B)$ preserves colimits (and hence admits a right adjoint).

(4) If $B$ is the limit of a cofiltered diagram of coherent schemes $B_{\alpha}$ with affine transition maps, then $\mathrm{SH}(B) \simeq \lim _{\alpha} \mathrm{SH}\left(B_{\alpha}\right)$ in the $\infty$-category of $\infty$-categories.

Proof (1) Let $E \in \mathrm{SH}(B)$ have components $E_{n} \in \mathrm{H}_{*}(B)$. By [16, Lemma 6.3.3.6],

$$
E \simeq \operatorname{colim}_{n \geq 0} \Sigma^{-\mathbb{A}^{n}} \Sigma^{\infty} E_{n},
$$

and each $\Sigma^{-\mathbb{A}^{n}} \Sigma^{\infty} E_{n}$ is clearly an iterated colimit of objects of the desired form.

(2) By Proposition C.7(1), $\mathrm{H}_{*}(B)$ is compactly generated by $X_{+}, X \in \mathrm{Sm}_{B}^{\mathrm{fp}}$. The object $S^{\mathbb{A}^{1}} \in \mathrm{H}_{*}(B)$ is compact, being a finite colimit of compact objects, and so the functor $\Omega^{\mathbb{A}^{1}}: \mathrm{H}_{*}(B) \rightarrow \mathrm{H}_{*}(B)$ preserves filtered colimits. The assertion now follows immediately from $(\mathrm{C}-11)$.

(3) We can assume that $B$ is coherent. By (1) and (2), $f^{*}$ sends a family of compact generators of $\mathrm{SH}(B)$ to compact objects in $\mathrm{SH}\left(B^{\prime}\right)$. By adjunction, $f_{*}$ preserves filtered colimits. Since $f_{*}$ preserves limits and both $\mathrm{SH}\left(B^{\prime}\right)$ and $\mathrm{SH}(B)$ are stable, it also preserves finite colimits.

(4) This follows from Proposition C.7(3) and (C-11).

Finally, we prove that $\mathrm{SH}(-)$ satisfies the proper base change theorem and related properties:

Proposition C.13 Let

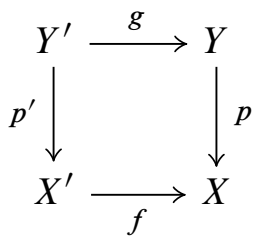

be a cartesian square of schemes where $p$ is proper.

(1) For every $E \in \mathrm{SH}(Y)$, the exchange transformation $E x_{*}^{*}: f^{*} p_{*} E \rightarrow p_{*}^{\prime} g^{*} E$ is an equivalence.

(2) For every $E \in \mathrm{SH}(Y)$ and $F \in \mathrm{SH}(X)$, the projector $P r_{*}^{*}: p_{*} E \wedge F \rightarrow p_{*}(E \wedge$ $\left.p^{*} F\right)$ is an equivalence.

(3) Suppose that $f$ is smooth. For every $E \in \mathrm{SH}\left(Y^{\prime}\right)$, the exchange transformation $E x_{\sharp *}: f_{\sharp} p_{*}^{\prime} E \rightarrow p_{*} g_{\sharp} E$ is an equivalence. 
Proof If $p$ is a closed immersion, all three statements follow easily from the gluing theorem. The argument of [3, Section 1.7.2] shows that the map $p_{\sharp} \rightarrow p_{*} \pi_{1 \sharp} \delta_{*}$ induced by $E x_{\sharp *}$ is an equivalence when $p$ is a projection $\mathbb{P}_{X}^{n} \rightarrow X$. The proof of [5, Lemma 2.4.23] then shows that (1-3) hold for such $p$. By Zariski descent, one immediately deduces (1-3) for $p$ projective. It remains to extend the results to $p$ proper.

(1) By Zariski descent, we can assume that $X$ and $X^{\prime}$ are coherent. Let $C: \mathrm{SH}(Y) \rightarrow$ $\mathrm{SH}\left(X^{\prime}\right)$ be the cofiber of the transformation $E x_{*}^{*}$. Since $\mathrm{SH}\left(X^{\prime}\right)$ is stable and compactly generated, it will suffice to show that $[K, C(E)]=0$ for every $E \in \mathrm{SH}(Y)$ and every $K \in \mathrm{SH}\left(X^{\prime}\right)$ compact. Fix $x: K \rightarrow C(E)$ and consider the poset $\Phi$ of closed subschemes $i: Z \hookrightarrow Y$ such that the image of $x$ in $\left[K, C\left(i_{*} i^{*} E\right)\right]$ is not zero. If $\left\{i_{\alpha}: Z_{\alpha} \hookrightarrow Y\right\}$ is a cofiltered diagram of closed subschemes of $Y$ with limit $i: Z \hookrightarrow Y$, it follows from (C-9) that $\operatorname{colim}_{\alpha} i_{\alpha *} i_{\alpha}^{*} \simeq i_{*} i^{*}$. Since the source and target of $E x_{*}^{*}$ preserve filtered colimits, the canonical map

$$
\underset{\alpha}{\operatorname{colim}} C\left(i_{\alpha *} i_{\alpha}^{*} E\right) \rightarrow C\left(i_{*} i^{*} E\right)
$$

is an equivalence. By compactness of $K$, we deduce that $\Phi$ is closed under cofiltered intersections. On the other hand, using Chow's lemma [2, XII,§7], the gluing theorem, and $(1,3)$ for $p$ projective, we easily verify that $\Phi$ does not have a minimal element. Hence, $\Phi$ is empty.

(2) Same proof as (1).

(3) Arguing as in (1) proves the result when $f$ is coherent. It also proves that $E x_{\sharp *} g^{*}$ is an equivalence, whence the result when $f$ is an open immersion. Without loss of generality, assume now that $X$ is coherent. Then $\mathrm{SH}\left(Y^{\prime}\right)$ is generated under colimits by the images of $h_{\sharp}$ where $h$ is the pullback of the inclusion of an open subscheme of $X^{\prime}$ which is coherent over $X$, so the general case follows.

By Nagata's compactification theorem [6] and Proposition C.13(3), we can apply Deligne's gluing theory and define the exceptional adjunction

$$
f_{!}: \mathrm{SH}(X) \rightleftarrows \mathrm{SH}(Y): f^{!}
$$

at the level of triangulated categories, for $f: X \rightarrow Y$ a separated morphism of finite type between coherent schemes. Following Cisinski-Déglise [5, Section 2], we then obtain the complete formalism of six operations for coherent schemes as described in Section 2.

Remark C.14 It is possible to define $\mathrm{SH}(-)$ as a contravariant functor from the category of schemes to the $\infty$-category of symmetric monoidal presentable $\infty$-categories. 
Using the $\infty$-categorical generalization of Deligne's gluing theory developed by Liu and Zheng [15], one can define the exceptional adjunction $f_{!} \dashv f^{!}$, the natural transformation $f_{!} \rightarrow f_{*}$ and all the exchange transformations and projectors involving exceptional functors, at the level of $\infty$-categories (for $f$ a separated morphism of finite type between coherent schemes). Since $\mathrm{SH}(-)$ is a Zariski sheaf, one can further define all this data for any morphism $f$ which is locally of finite type. Once this is done, the conventions set at the end of Section 1 can be ignored altogether and "separated of finite type" can be replaced everywhere by "locally of finite type".

\section{References}

[1] M Artin, A Grothendieck, J-L Verdier, SGA 4: Théorie des topos et cohomologie étale des schémas, Tome 1, Lect. Notes Math. 269, Springer, Berlin (1972)

[2] M Artin, A Grothendieck, J-L Verdier, SGA 4: Théorie des topos et cohomologie étale des schémas, Tome 3, Lect. Notes Math. 305, Springer, Berlin (1973) MR0354654

[3] J Ayoub, Les six opérations de Grothendieck et le formalisme des cycles évanescents dans le monde motivique, I, Astérisque 315, Soc. Math. France

[4] J Ayoub, La réalisation étale et les opérations de Grothendieck, Ann. Sci. Éc. Norm. Supér. 47 (2014) 1-141 MR3205601

[5] D-C Cisinski, F Déglise, Triangulated categories of mixed motives (2012) arXiv: 0912.2110v3

[6] B Conrad, Deligne's notes on Nagata compactifications, J. Ramanujan Math. Soc. 22 (2007) 205-257 MR2356346

[7] A Dold, Lectures on algebraic topology, Classics in Mathematics, Springer, Berlin (1995) MR1335915

[8] A Dold, D Puppe, Duality, trace and transfer, Trudy Mat. Inst. Steklov. 154 (1983) 81-97 MR733829 In Russian; translated in Proceedings of the Steklov Institute of Mathematics 154 (1984) 85-103

[9] W Fulton, Intersection theory, 2nd edition, Ergeb. Math. Grenzgeb. 2, Springer, Berlin (1998) MR1644323

[10] W-D Geyer, G Harder, M Knebusch, W Scharlau, Ein Residuensatz für symmetrische Bilinearformen, Invent. Math. 11 (1970) 319-328 MR0283005

[11] A Grothendieck, SGA 5: Cohomologie $\ell$-adique et fonctions L, Lect. Notes Math. 589, Springer, Berlin (1977) MR0463174

[12] A Grothendieck, Dieudonneé, EGA IV: Étude locale des schémas et des morphismes de schémas, Publ. Math. IHÉS 32, IHÉS (1967) 
[13] M Hoyois, From algebraic cobordism to motivic cohomology arXiv:1210.7182To appear in J. Reine Angew. Math.

[14] P Hu, On the Picard group of the stable $\mathbb{A}^{1}$-homotopy category, Topology 44 (2005) 609-640 MR2122218 Appendix A jointly written with I Kriz

[15] Y Liu, W Zheng, Gluing restricted nerves of $\infty$-categories (2014) arXiv: $1211.5294 \mathrm{v} 3$

[16] J Lurie, Higher topos theory, Annals Math. Studies 170, Princeton Univ. Press (2009) MR2522659

[17] J P May, The additivity of traces in triangulated categories, Adv. Math. 163 (2001) 34-73 MR1867203

[18] J Milnor, D Husemoller, Symmetric bilinear forms, Ergeb. Math. Grenzgeb. 73, Springer, New York (1973) MR0506372

[19] F Morel, $\mathbb{A}^{1}$-algebraic topology, from: "Proceedings of the International Congress of Mathematicians, Vol. II", (M Sanz-Solé, J Soria, J L Varona, J Verdera, editors), Eur. Math. Soc., Zürich (2006) 1035-1059 MR2275634

[20] F Morel, $\mathbb{A}^{1}$-algebraic topology over a field, Lect. Notes Math. 2052, Springer, Heidelberg (2012) MR2934577

[21] F Morel, V Voevodsky, $\mathbf{A}^{1}$-homotopy theory of schemes, Inst. Hautes Études Sci. Publ. Math. (1999) 45-143 MR1813224

[22] Y A Nisnevich, The completely decomposed topology on schemes and associated descent spectral sequences in algebraic $K$-theory, from: "Algebraic $K$-theory: connections with geometry and topology", (J F Jardine, V P Snaith, editors), NATO Adv. Sci. Inst. Ser. C Math. Phys. Sci. 279, Kluwer, Dordrecht (1989) 241-342 MR1045853

[23] J Riou, Dualité de Spanier-Whitehead en géométrie algébrique, C. R. Math. Acad. Sci. Paris 340 (2005) 431-436 MR2135324

[24] M Robalo, Noncommutative motives I: A universal characterization of the motivic stable homotopy theory of schemes (2013) arXiv:1206.3645v3

[25] W Scharlau, Quadratic reciprocity laws, Journal of Number Theory 4 (1972) 78-97 MR0292753

[26] J-P Serre, Corps locaux, Hermann, Paris (1968) MR0354618

[27] R W Thomason, T Trobaugh, Higher algebraic K-theory of schemes and of derived categories, from: "The Grothendieck Festschrift, Vol. III", (P Cartier, L Illusie, N M Katz, G Laumon, Y I Manin, K A Ribet, editors), Progr. Math. 88, Birkhäuser, Boston (1990) 247-435 MR1106918

[28] V Voevodsky, Motivic cohomology with Z/2-coefficients, Publ. Math. Inst. Hautes Études Sci. (2003) 59-104 MR2031199 
[29] V Voevodsky, Homotopy theory of simplicial sheaves in completely decomposable topologies, J. Pure Appl. Algebra 214 (2010) 1384-1398 MR2593670

Department of Mathematics, Northwestern University

2033 Sheridan Road, Evanston, IL 60208, USA

hoyois@math.northwestern.edu

http://math.northwestern.edu/ hoyois/

Received: 1 November 2013 Revised: 13 June 2014 